\title{
Nonclassical Resident Macrophages Are Important Determinants in the Development of Myocardial Fibrosis
}

\author{
Alec Falkenham, ${ }^{\star}$ Roberto de Antueno, ${ }^{\dagger}$ Nicole Rosin, ${ }^{*}$ Devin Betsch, ${ }^{\dagger}$ Timothy D.G. Lee, ${ }^{* \dagger}{ }^{\star \dagger}$ Roy Duncan, ${ }^{\dagger}$ and \\ Jean-Francois Légaré* ${ }^{\star \ddagger}$
}

From the Departments of Pathology, ${ }^{*}$ Microbiology and Immunology, ${ }^{\dagger}$ and Surgery, ${ }^{\ddagger}$ Dalhousie University, Halifax, Nova Scotia, Canada

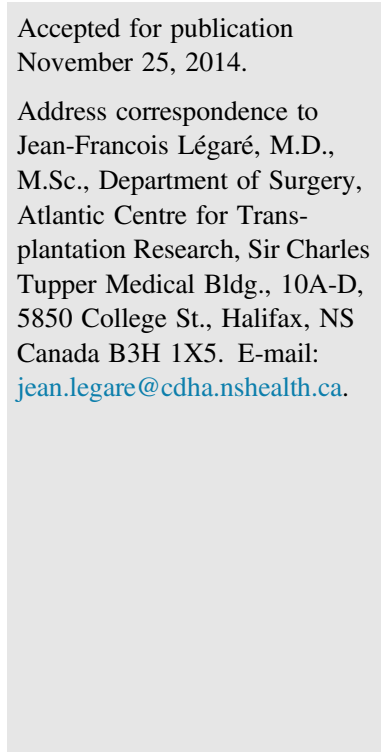

Accepted for publication November 25, 2014

Address correspondence to Jean-Francois Légaré, M.D. M.Sc., Department of Surgery, Atlantic Centre for Transplantation Research, Sir Charles Tupper Medical Bldg., 10A-D, 5850 College St., Halifax, NS Canada B3H 1X5. E-mail: jean.legare@cdha.nshealth.ca.

\begin{abstract}
Macrophages are increasingly recognized as a potential therapeutic target in myocardial fibrosis via interactions with fibroblasts. We have characterized macrophage depletion and inhibition of nonclassical macrophage migration, in addition to direct interactions between nonclassical macrophages and fibroblasts in angiotensin II (AngII)-mediated, hypertensive myocardial fibrosis. Macrophage depletion was achieved by daily i.v. clodronate liposomes ( -1 day to +3 days) during AngII infusion. $C \times 3 \mathrm{Cr}^{-1-}$ mice were used to inhibit nonclassical macrophage migration. Macrophage phenotype (F4/80, CD11b, Ly6C) was characterized by immunofluorescence and flow cytometry. Collagen was assessed by Sirius Red/Fast Green. Quantitative real-time RT-PCR was performed for transcript levels. AngII/wild-type (WT) mice displayed significant infiltrate and fibrosis compared with saline/WT, which was virtually ablated by clodronate liposomes independent of hypertension. In vitro data supported M2 macrophages promoting fibroblast differentiation and collagen production. AngII/C $\times 3 \mathrm{Cr}^{-/-}$mice, however, significantly increased macrophage infiltrate and fibrosis relative to AngII/WT. AngII/C $\times 3 \mathrm{Cr}^{-/-}$mice also showed an M1 phenotypic shift relative to WT mice in, which the predominant phenotype was Ly6C ${ }^{\text {low }}, \mathrm{CD}_{206}{ }^{+}(\mathrm{M} 2)$. Myocardial IL-1 $\beta$ was significantly upregulated, whereas transforming growth factor $\beta$ down-regulated with this M1 shift. We demonstrated that infiltrating macrophages are critical to AngII-mediated myocardial fibrosis by preventing the development of fibrosis after liposomal depletion of circulating monocytes. Our findings also suggest that some macrophages, namely $\mathrm{M} 2$, may confer a protective myocardial environment that may prevent excessive tissue injury. (Am J Pathol 2015, 185: 927-942; http://dx.doi.org/10.1016/j.ajpath.2014.11.027)
\end{abstract}

Myocardial fibrosis is a common feature in cardiovascular diseases and is suspected to be the final pathway in the development of heart failure. ${ }^{1}$ The characteristic feature of myocardial fibrosis is the abnormal accumulation of extracellular matrix (ECM) proteins. As ECM proteins accumulate, the heart stiffens, and myocardial dysfunction develops that can ultimately culminate in heart failure. In the United States alone, almost 6 million people are afflicted with heart failure, which contributes to an estimated $>300,000$ deaths per year, emphasizing the importance of understanding the mechanisms underlying the development of myocardial fibrosis. ${ }^{2}$

Fibroblasts are described as the effector cell responsible for ECM deposition; however, there is increasing evidence that macrophages may be key orchestrators of fibrosis through their interactions with fibroblasts. ${ }^{3-8} \mathrm{An}$ evolving understanding of macrophage plasticity has added complexity to the relation between macrophages and fibrosis. We now know that macrophages are a heterogeneous population capable of proinflammatory (classical), regulatory/ anti-inflammatory (nonclassical), and intermediate phenotypes. In humans, these populations represent a wide spectrum of potential differentiation and activation state and are characterized by their expression of CD14, CD16, C-C chemokine receptor type 2 (CCR2), and CX3C chemokine receptor 1 (CX3CR1), among other markers. On one end of

Supported by Canadian Institute of Health Research grant 324531 (J.F.L.); a CIHR doctoral scholarship, an NSHRF doctoral scholarship, Killam Laureate, and a BrightRed Graduate Research Award (A.F.).

Disclosures: None declared. 
the spectrum, the classical or M1 population, is CD14 ${ }^{\text {high }}$, $\mathrm{CD} 16^{-}, \mathrm{CCR} 2^{\text {high }}$, and CX3CR $1^{\text {low }} \cdot{ }^{9,10}$ Conversely, on the other end of the spectrum, the nonclassical or M2 population, is $\mathrm{CD} 14^{\text {low }}, \mathrm{CD} 16^{\text {low-high }}, \mathrm{CCR} 2^{\text {low }}$, and CX3CR $1^{\text {high }} \cdot 9,10$ This chemokine receptor expression is mirrored in mice; however, mice do not express CD16, and the M1 to M2 transition is characterized by the loss of Ly6C expression. 9,10 Thus, the consistency of the M1/M2 paradigm between mice and humans supports the use of mouse models to characterize the roles of monocyte and macrophage subsets in disease.

Although M1 macrophage-mediated inflammation can worsen tissue injury and exacerbate fibrotic responses, M2 macrophages can also produce anti-inflammatory and profibrotic factors such as IL-10, transforming growth factor $\beta$ (TGF- $\beta$ ), connective tissue growth factor (CTGF), and plateletderived growth factor to directly promote fibroblast proliferation and ECM production. ${ }^{6,11,12}$ These macrophage activation states do not exist exclusively from one another. Rather, the contribution of macrophage subsets to fibrosis is part of a dynamic response determined by the demands of healing tissues and characterized by differential monocyte recruitment and the ability to shift between functional phenotypes.

Macrophage phenotype change during fibrotic responses is suggested in a number of fibrosis models, including liver, lung, and kidney; however, the evidence in the myocardium is only suggestive and limited to ischemic injury. ${ }^{3,12-14}$ Work in our laboratory by using a hypertension model of myocardial fibrosis has shown that myocardial fibrosis is preceded by a mass influx of circulating mononuclear cells characteristic of macrophages, expressing markers such as $\mathrm{ED} 1, \mathrm{CD} 1 \mathrm{~b}$, and F4/80 ${ }^{15,16}$ Infiltrating monocytes can dramatically expand the macrophage population in the heart.

Recent findings have also suggested that an important resident macrophage population exists in the myocardium, thus providing an additional source of macrophage-fibroblast interactions, but their role in myocardial fibrosis is unknown. ${ }^{17}$ These new knowledge could mean that monocytes and macrophages may represent important therapeutic targets for myocardial fibrosis rather than targeting the final effector cell, the fibroblast. However, elucidating the contributions of the different macrophage populations and specific subsets in myocardial fibrosis needs to be better understood and is a necessary step toward macrophage-dependent therapies. Liposomal monocyte depletion and chemokine receptor knockouts are valuable tools for characterizing the contribution of macrophages subsets. ${ }^{18-20}$

Here, we use the well-established angiotensin II (AngII) model to study myocardial fibrosis, which depends on sustained hypertension and renin angiotensin aldosterone system activation as is seen clinically in patients. ${ }^{15,16,21-23}$ The AngII model is characterized by significant macrophage infiltrate, a profibrotic milieu, and significant ECM deposition without the loss of healthy tissue as seen in ischemic injury. ${ }^{15,16,24}$ Furthermore, our work with $\mathrm{Ccr} 2^{-/-}$ mice in this model suggests that the nonclassical $\mathrm{Cx} 3 \mathrm{crl}$ pathway may be important to nonischemic myocardial healing. ${ }^{21}$ This model offers the unique opportunity to study myocardial fibrosis without conflicting ischemia that is seen after myocardial infarction and to focus the depletion to the fibrotic stage of the response.

Here, we have characterized early macrophage accumulation and the effects of monocyte depletion on the development of AngII-mediated myocardial fibrosis. Specifically, we examined the effects of liposome monocyte depletion on cellular infiltrate, the profibrotic environment, and the development of myocardial fibrosis. In addition, we are the first group to characterize myocardial healing in the absence of $C x 3 \mathrm{crl}$ signaling, the outcomes of which could have important therapeutic implications.

\section{Materials and Methods}

\section{Mice}

Animal experiments were performed in accordance with the Canadian Council on Animal Care and approved by the Dalhousie University Committee on Laboratory Animals. Male C57BL/6 mice [8 to 10 weeks old; wild-type (WT)] were purchased from The Jackson Laboratory (Bar Harbor, ME), and male $\mathrm{C} x 3 \mathrm{Crl}^{-/-}$mice (8 to 10 weeks old) on a C57BL/6 background were acquired through a material transfer agreement with Taconic Farms (Germantown, NY) ${ }^{25}$ Mice were housed in the Carleton Animal Care Facility at Dalhousie University and were provided food and water ad libitum for 1 week before beginning experimentation.

\section{Myocardial Fibrosis Model}

Osmotic minipumps (Alzet, Palo Alto, CA) were implanted as previously described. ${ }^{21}$ Mice were randomly assigned treatments of vehicle (saline) or $2.8 \mathrm{mg} / \mathrm{kg}$ per day AngII (SigmaAldrich, Oakville, ON, Canada). The pumps remained in for 3 days, during which time, the mice were observed for morbidity. Mice were weighed, and blood pressure measurements were taken with the Coda2 noninvasive tail cuff system (Kent Scientific, Torrington, CT) for a minimum of five measurements per mouse. At the time of harvest, hearts were extracted, flushed with saline, and weighed.

\section{Liposomes}

Liposomes were generated as previously described, with modification. $^{26}$ In brief, egg phosphotidyl choline and cholesterol (82.4:15.6\% $\mathrm{mol} / \mathrm{L}$ ratio, respectively) in chloroform were added to sterile pear-shaped flasks and placed under rotary evaporation until excess chloroform was removed to dryness, and a uniform film developed. Lipidsoluble DiO was added for liposome binding and uptake studies. The film was subsequently hydrated with either 300 $\mathrm{mg} / \mathrm{mL}$ clodronate (Sigma-Aldrich) or saline, and liposomes were left to swell for 2 hours at room temperature. Liposomes were then sonicated $(4$ minutes at $55 \mathrm{~Hz})$ and washed of free clodronate by centrifuging four to five times at 
$24,000 \times g$ for 5 minutes at $10^{\circ} \mathrm{C}$. Before injection, liposomes were passed through a $3.0-\mu \mathrm{m}$ pore filter (Millipore, Billerica, MA). The final concentration of lipid in the liposomes was $33.07 \mathrm{mmol} / \mathrm{L}$. Liposomes were injected in $100-\mu \mathrm{L}$ doses via tail vein every 24 hours, beginning at -1 day before AngII infusion.

\section{Leukocyte Quantification}

At 3 days, mice were sacrificed, and blood was collected for smears. Smears were stained with Giemsa (GS500; SigmaAldrich) for nuclear morphology, and leukocyte populations were counted by a blind observer (A.F.). A total of 350 cells were characterized per slide.

\section{Immunohistochemistry and Immunofluorescence}

Hearts were processed in $10 \%$ buffered formalin overnight and paraffin-embedded. Serial sections $(5 \mu \mathrm{m})$ were stained for histologic analysis. Basic myocardial histology and cellular infiltrate were assessed with heart sections stained with hematoxylin and eosin. The area of the heart affected was calculated as previously described. ${ }^{21-23}$ Fibrotic deposition was examined with heart sections stained with Picosirius Red (SR) and the counterstain Fast Green (FG). Collagen content was semiquantified by photographing representative SR/FG whole heart sections at $\times 5$ magnification. With the use of Photoshop CS6 (Adobe Systems, San Jose, CA), red pixels were positively selected and summed for a total number of red (collagen) pixels. Subsequently, nonbackground pixels were summed for the total heart pixels. Collagen pixels were divided by the total heart pixels to provide a semiquantitative measurement of the percentage of collagen content in the heart. All tissues were processed simultaneously for SR/FG, and the same red color palette was used to select red pixels.

For frozen sections, anesthetized mice were perfused with saline, followed by $4 \%$ paraformaldehyde. Hearts were extracted and placed in $4 \%$ paraformaldehyde overnight. The hearts were transferred to $30 \%$ sucrose, and $10-\mu \mathrm{m}$ sections were cut on a cryostat. Immunofluorescence was performed on both frozen heart sections and fixed cocultures for CD11b (AbD Serotec, Raleigh, NC), collagen type-I (Rockland, Gilbertsville, PA), F4/80 (Abcam, Cambridge, MA), $\alpha$-smooth muscle cell actin ( $\alpha$-SMA; Sigma-Aldrich), CD206 (AbD Serotec), CX3CR1 (Abcam), and CD107b (AbD Serotec). In brief, sections were permeabilized with $0.1 \%$ Triton X-100/phosphate-buffered saline (PBS), blocked with $5 \%$ bovine serum albumin/PBS, and then incubated with primary antibodies overnight at $4^{\circ} \mathrm{C}$. Sections were then incubated with Alexa Fluor 488, Alexa Fluor 555, Alexa Fluor 647, or biotin-conjugated secondary antibodies. Biotin-conjugated antibodies were linked to avidin and developed with diaminobenzidine. In fluorescence, nuclei were stained with DAPI. Slides were visualized with Axiovert 200M (Carl Zeiss Inc., Thornwood, NY) and photographed with an Orca R2 camera (Hamamatsu Corporation, Bridgewater, NJ).

\section{Coculture}

The macrophage-fibroblast coculture was conducted as previously described with modification. ${ }^{5,27}$ Bone marrowderived macrophages (BMDMs) were generated as previously described. ${ }^{28}$ In brief, mice were anesthetized and then sacrificed, and the femurs and tibias were isolated. Cells were flushed from the marrow by using a 30-gauge needle attached to a syringe that contained DMEM (Dulbecco's Modified Eagle Media) complete (DMEM-C: DMEM, L-glutamine, Pen Strep, $10 \%$ fetal bovine serum). After washes and cell straining, BMDMs were resuspended in DMEM-C with $10 \%$ L929 conditioned medium and plated in T75 flasks. Media were replaced on day 5. On day 7, the BMDMs were lifted with $0.25 \%$ trypsin, washed with DMEM-C, counted, and replated-plated in 12-well plates at a density of $2 \times 10^{5}$ per well. Additional macrophages were screened for CD11b and Ly6C expression by using flow cytometry as described in Immunohistochemistry and Immunofluorescence. NIH/3T3 cells (ATCC, Manassas, VA) served as fibroblasts in monocultures and cocultures. NIH/ $3 \mathrm{~T} 3$ cells were maintained in DMEM-C until lifted with $0.25 \%$ trypsin, washed with DMEM-C, counted, and replated at $2 \times 10^{5}$ per well. Monocultures and cocultures were allowed to sit down overnight in DMEM-C before being serum-starved for 24 hours. The next day, the media were replaced with DMEM-C, and cells were incubated for 48 hours. At 48 hours, the supernatant fluid was removed, and the cells were washed with PBS, then fixed in 4\% paraformaldehyde. The cells were immunofluorescently labeled as described in Immunohistochemistry and Immunofluorescence, and wells were read for fluorescence by using a Tecan infinite M200 Pro (Tecan, Männedorf, Germany) plate reader. $\alpha$-SMA expression was standardized to DAPI fluorescence intensity for cocultures and fibroblast monocultures. The coculture expression was then calculated relative to fibroblast monoculture expression. Immunofluorescence and flow cytometry were used to quantify cell purity.

\section{Isolation of Cells from Myocardium}

Hearts from WT and knockout mice infused with AngII for 3 days were harvested under sterile conditions and were used for cell isolation as previously described with modification. ${ }^{16,21}$ In brief, hearts were mechanically and enzymatically digested in a collagenase solution $(0.652 \mathrm{mg} / \mathrm{mL}$ collagenase II; Cedarlane Laboratories, Ltd., Burlington, ON, Canada) in DMEM-C at $37^{\circ} \mathrm{C}$, with agitation for 45 minutes. Cell isolates were twice washed in DMEM-C and purified over a Percoll gradient (30\% and 70\%). Cells were then counted and used for flow cytometric analysis. Cells were washed with fluorescence-activated cell sorting buffer (Dulbecco PBS, $1 \%$ bovine serum albumin, $0.1 \% \mathrm{NaN}_{3}$ ) and incubated with antibodies anti-CD11b-allophycocyanin 
(eBioscience, San Diego, CA), anti-F4/80-peridinin chlorophyll protein complex cyanine 5.5 (Biolegend, San Diego, CA), and anti-Ly6C-phycoerythrin (Biolegend). After incubation, cells were washed twice with fluorescenceactivated cell sorting buffer and fixed with $1 \%$ formalin/ fluorescence-activated cell sorting buffer. Isotype control (Santa Cruz Biotechnology, Santa Cruz, CA) was used for negative gating. Data were acquired with a BD FACSCalibur (BD Biosciences, San Jose, CA) and were analyzed with WinList 3D (Verity Software, Topsham, ME). Macrophage gating was performed by gating on cells with the use of forward and side scatter (SSC), which was then applied to a CD11b $\times$ F4/80 dot plot. The F4/80 ${ }^{+}$events were gated and applied to a CD11b $\times$ Ly6C dot plot for the characterization Ly6C mean fluorescent index of mature and immature macrophage populations in the myocardium. When F4/80 labeling was not performed, CD11b $\times$ SSC was used to distinguish polymorphonuclear cells (SSC ${ }^{\text {high }}$ ) and monocyte-derived cells (SSC ${ }^{\text {low-mid }}$ ).

\section{Echocardiography}

Before echocardiography, mice were anesthetized, and the fur was removed with hair removal cream (Nair; Church \& Dwight Co., Inc., Ewing, NJ). Images and recordings were acquired on a Vivid 7 Ultrasound (GE Healthcare, Chalfont St. Giles, UK). Short-axis M-mode measurements for left ventricular posterior wall and intraventricular septal wall thicknesses were performed in Photoshop CS6 (Adobe Systems).

\section{Relative Quantitative Real-Time PCR}

RNA isolation, cDNA generation, and quantitative real-time PCR were performed as previously described. ${ }^{15,21,22}$ Primers were designed against mRNA sequences of chemokine (C-X3-C motif) ligand 1 (CX3CL1; forward, 5'-GCAACCCCACCCCTTATCAA-3'; reverse, 5'-CTTGCCAGCCCTCAGAATCA-3'), TGF- $\beta$ (forward, 5'-CTCTTGAGTCCCTCGCATCC-3'; reverse, 5'-GGTCTCCCAAGGAAAGGTAGG- $3^{\prime}$ ), CTGF (forward, $5^{\prime}$-TAGAGCAGGTCTGTCTGCAAG-3'; reverse, $5^{\prime}$-TCAACCTCAGACACTGGTTTC- $3^{\prime}$ ), IL-1 $\beta$ (forward, 5'-TCCTCGGCCAAGACAGGTCGCT-3'; reverse, 5'-CCCCCACACGTTGACAGCTAGGT-3'), collagen $\alpha 1 \alpha$ (forward, $5^{\prime}$-CAACAGTCGCTTCACCTACAGC$3^{\prime}$; reverse, $5^{\prime}$-GTGGAGGGAGTTTACACGAAGC- $3^{\prime}$ ), and tumor necrosis factor $\alpha$ (forward, $5^{\prime}$-TCTCATGCACCACCATCAAGGACT-3'; reverse, 5'-ACCACTCTCCCTTTGCAGAACTCA-3'). Expression was normalization to the 18S (forward, 5'-TCAACTTTCGATGGTAGTCGCCGT-3'; reverse, 5'-TCCTTGGATGTGGTAGCCGTTTCT-3') ribosomal gene by using the Pfaffl method.

\section{Statistical Analysis}

Results are expressed as means \pm SEM. Multiple group comparisons were performed by one-way analysis of variance followed by Bonferroni post-test for comparing means. One-sample $t$-tests were used for relative comparisons between experimental groups and standardized controls.

\section{Results}

Macrophages Are Present in the Myocardium after 3 Days of AngII Infusion

Previous work in our laboratory which used a wellestablished AngII infusion model of hypertension and hypertrophy has demonstrated that bone marrow-derived leukocyte infiltration precedes the development of myocardial fibrosis. ${ }^{15,16,21-23}$ Furthermore, we have established that infiltration starts by 1 day and peaks at 3 days of AngII infusion, thus making it an important time point to characterize infiltrating cells.

Consistent with previous findings, most infiltrating cells in the AngII-exposed myocardium were mononuclear (Figure 1A). ${ }^{15,20-22}$ We then further characterized the infiltrating cells by using flow cytometry and immunohistochemistry. In saline controls, $2.58 \% \pm 0.01 \%$ of nonmyocytes isolated from the heart expressed the $\mathrm{CD}_{11} \mathrm{~b}^{+} \mathrm{SSC}^{\text {low }}$ macrophage phenotype, likely representing resident cardiac macrophages, as recently described (Figure 1, B and D). ${ }^{17,29,30}$ After AngII infusion, the percentage of cells expressing the macrophage phenotype significantly increased to $19.33 \% \pm 1.53 \%$, a 7.49 -fold increase over saline controls $(P<0.0001)$ (Figure 1, C and D). Consistent with nonclassical macrophages, cells expressing the chemokine receptor CX3CR $1^{+}$were observed in the areas of macrophage accumulation (Figure 1E). These macrophages were further characterized by flow cytometry for Ly6C expression.

In both the saline- (Figure 1F) and AngII-exposed (Figure $1 \mathrm{G}$ ) myocardia, the $\mathrm{CD}_{11 \mathrm{~b}^{+}} \mathrm{SSC}^{\text {low }}$ population displayed a shift toward Ly6C ${ }^{\text {low }}$ expression, indicative of a nonclassical phenotype. When quantified in the AngIIinfused group, the $\mathrm{Ly} 6 \mathrm{C}^{\text {low }}$ population represented $54.56 \% \pm 1.93 \%$, whereas the Ly6 $\mathrm{C}^{\text {mid }}$ and $\mathrm{Ly} 6 \mathrm{C}^{\text {high }}$ groups comprised $27.46 \% \pm 0.74 \%$ and $18.05 \% \pm 1.58 \%$, respectively $(P<0.001)$ (Figure $1 \mathrm{H})$. Favoring the Ly6C $\mathrm{C}^{\text {low }}$ CX3CR $1^{+}$macrophage phenotype is consistent with previous work in which $\mathrm{Ccr} 2^{-/-}$mice did not demonstrate a reduction in early cellular infiltrate or fibrosis in the myocardium early after AngII exposure. ${ }^{21}$ Furthermore, our previous work with green fluorescent protein bone marrow chimeras indicated that these areas of cellular infiltrate are predominantly derived from circulating monocytes. ${ }^{15}$ It followed that these macrophages could be depleted with i.v. liposomes.

\section{Macrophages Can Be Targeted with Liposomes in Circulation}

Circulating monocyte targeting was evaluated by administering fluorescent, $\mathrm{DiO}^{+}$liposomes to mice during AngII 

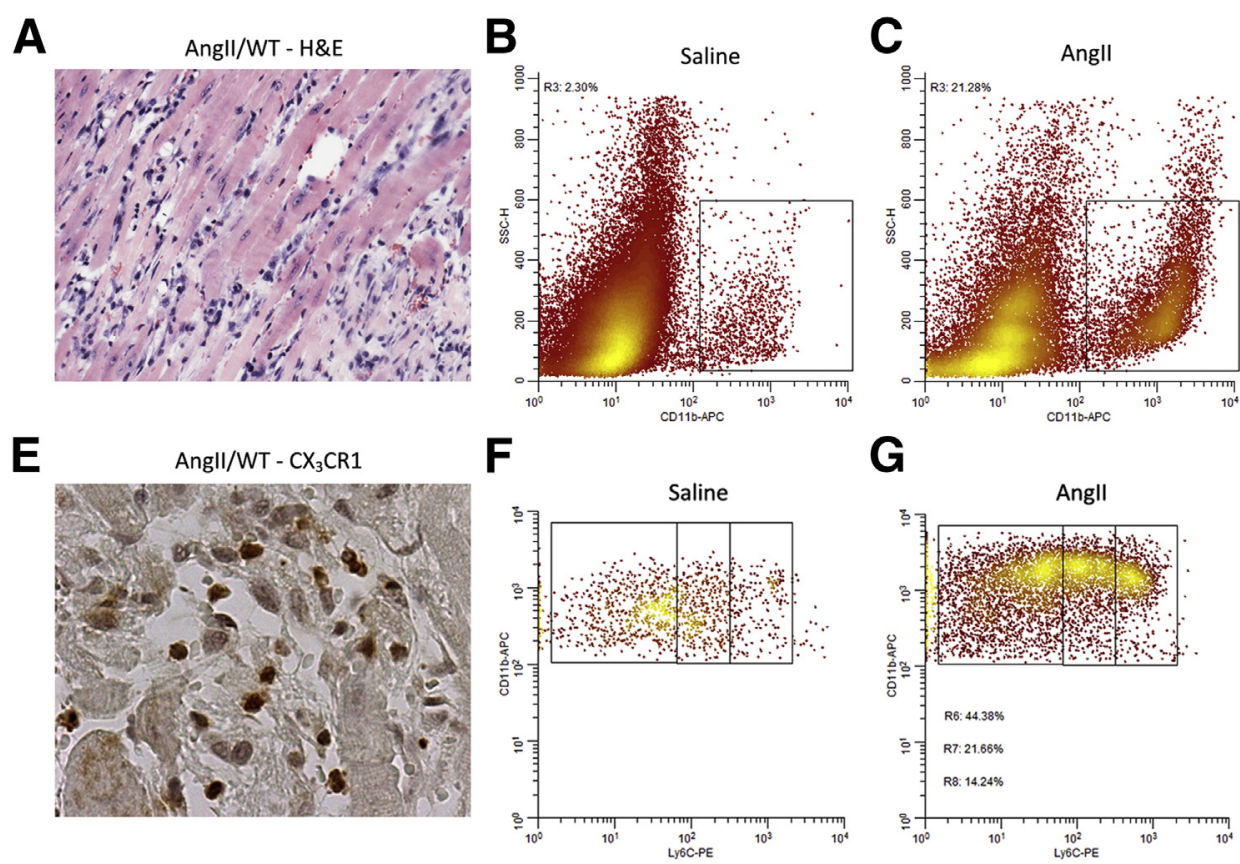

G

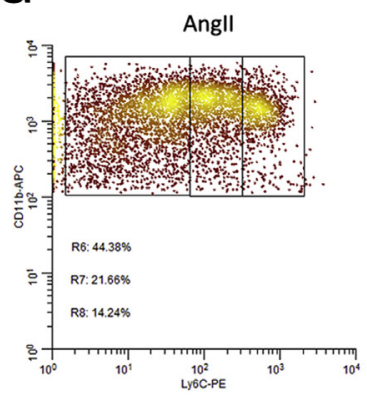

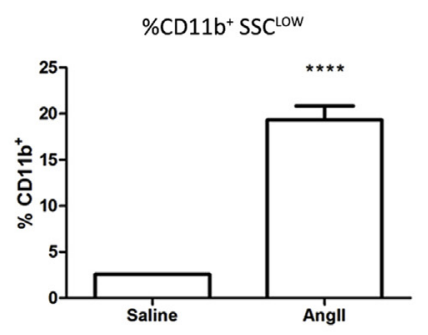

H

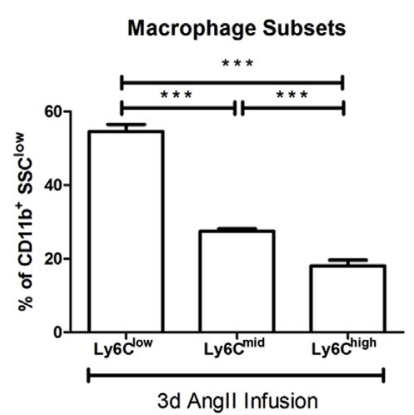

Figure 1 AngII leads to a macrophage influx in the heart. A: Representative image of myocardium stained with H\&E from an animal that received AngII. B and C: Flow cytometry of overall cardiac macrophage (resident + infiltrating) populations in saline control (B) and AngII-exposed (C) hearts as defined by $\mathrm{CD}_{11} \mathrm{~b}^{+} \mathrm{SSC}^{\text {low }}$ gates (boxed areas). D: Quantification of $\mathrm{CD}_{11 \mathrm{~b}^{+}} \mathrm{SSC}^{\mathrm{low}}$ macrophage populations of saline- and AngII-exposed myocardia. E: Representative immunohistochemistry for CX3CR1 in myocardium from an AngII/WT animal that received AngII. F and G: Representative flow cytometry on CD11 $b^{+}$SSC ${ }^{\text {low }}$ populations from WT mice that received saline $(\mathbf{F})$ and AngII (G). H: Semiquantitative distribution of Ly6C expression on CD11b ${ }^{+}$SSClow populations. Data are expressed as means \pm SEM. $n=4$ to 8 . ${ }^{* * *} P<0.001,{ }^{* * *} P<0.0001$. Original magnification: $\times 20$ (A); $\times 63$ (E). AngII, angiotensin II; APC, allophycocyanin; CX3CR1, CX3C chemokine receptor 1; H\&E, hematoxylin and eosin; PE, phycoerythrin; SSC, side scatter; WT, wild-type; 3d, 3-day.

infusion. Liposome administration was initiated 1 day before implanting an osmotic minipump that contained AngII to saturate circulating monocytes with liposomes. Mice were sacrificed after 3 days of AngII infusion, and frozen sections of heart were characterized by immunofluorescence. In a representative section of myocardium, a population of $\mathrm{CD}_{11} \mathrm{~b}^{+}$cells (Figure $2 \mathrm{~A}$ ) were observed in areas of collagen deposition (Figure 2A). In addition, punctate green fluorescence was observed in these same areas, consistent with $\mathrm{DiO}^{+}$liposomes (Figure 2A). Cell nuclei were labeled with DAPI (Figure 2A). Immunofluorescence staining confirmed the colocalization of liposomes in CD11b-expressing cells (Figure 2B). Liposomes were not observed in nonmacrophages, supporting i.v. phagocytosis
A
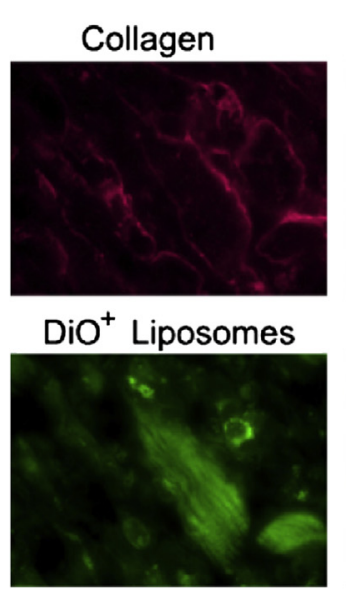

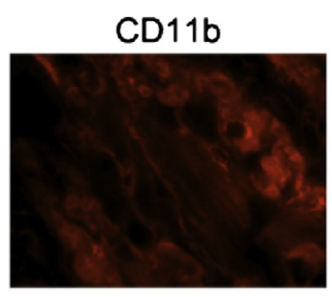

DAPI

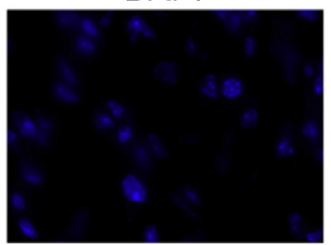

B

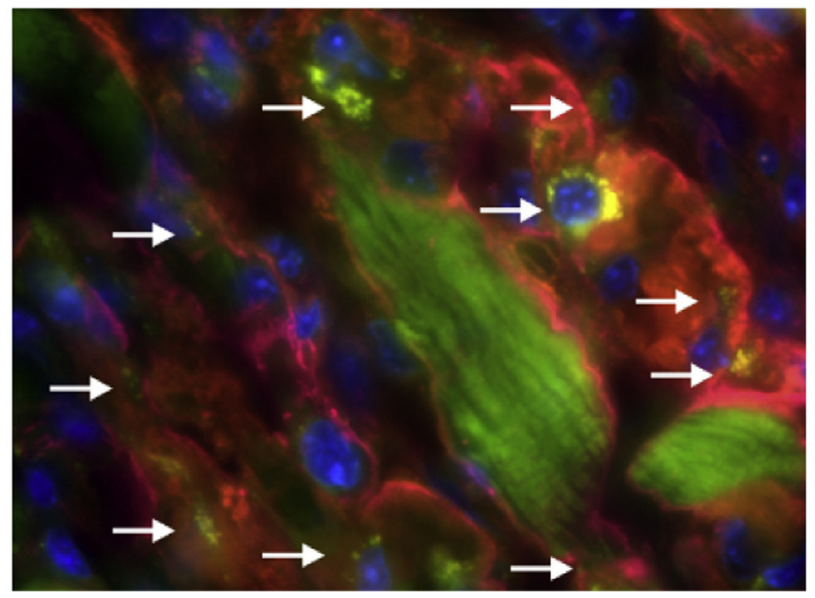

Figure 2 Cardiac macrophages in the AngII-exposed myocardium can be targeted intravenously by liposomes. Fluorescent (Di0 ${ }^{+}$) liposome uptake by macrophages in areas of collagen deposition from representative myocardial sections. A: Single labels for collagen type-I, CD11b, Di0 liposomes, and nuclei (DAPI). B: Overlay of individual channels demonstrating punctate yellow fluorescence, characteristic of colocalization with $\mathrm{DiO}^{+}$liposomes. Arrows indicate examples of colocalization. Original magnification, $\times 100$. AngII, angiotensin II. 


\section{A}

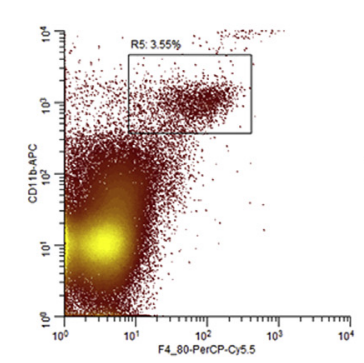

$\mathbf{E}$

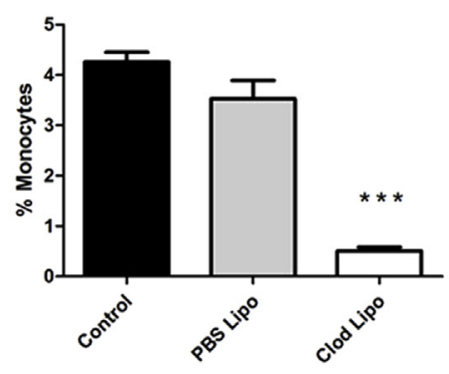

C

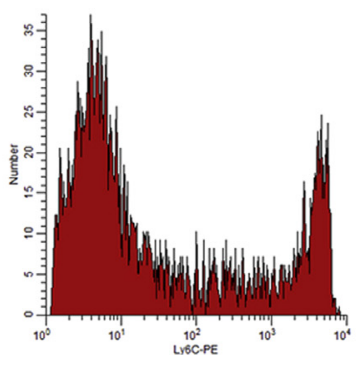

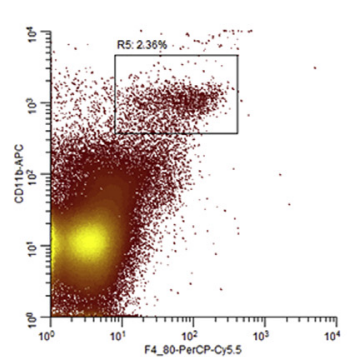

D

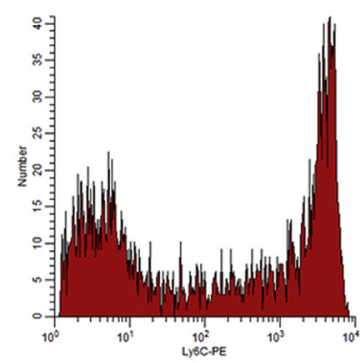

$\mathbf{F}$

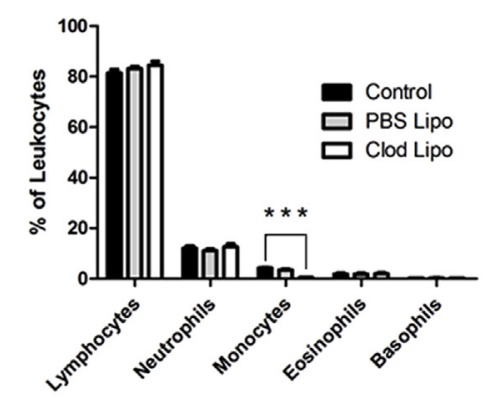

Angll / WT
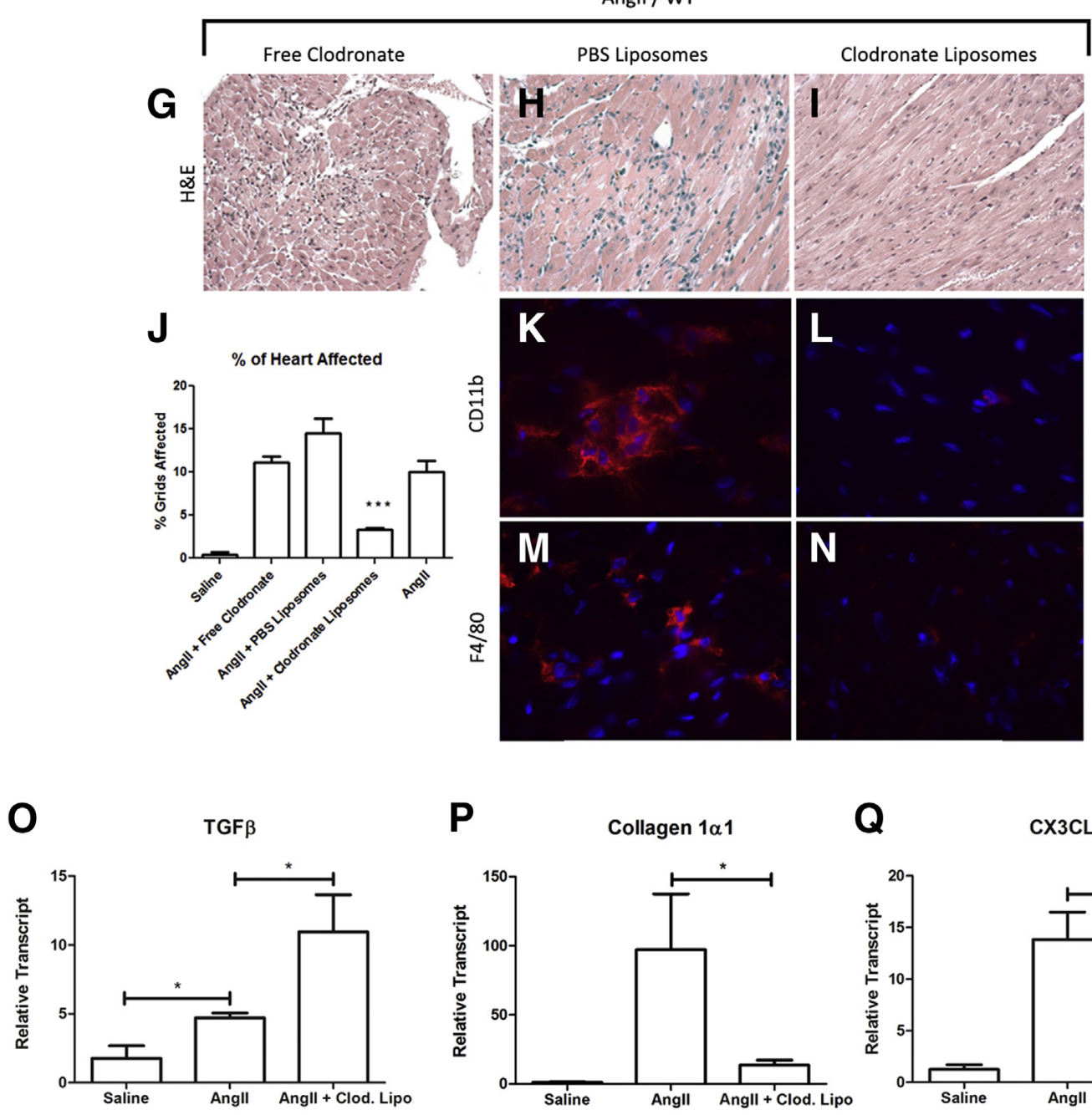

$\mathbf{P}$

Collagen $1 \alpha 1$

Q

CX3CL1
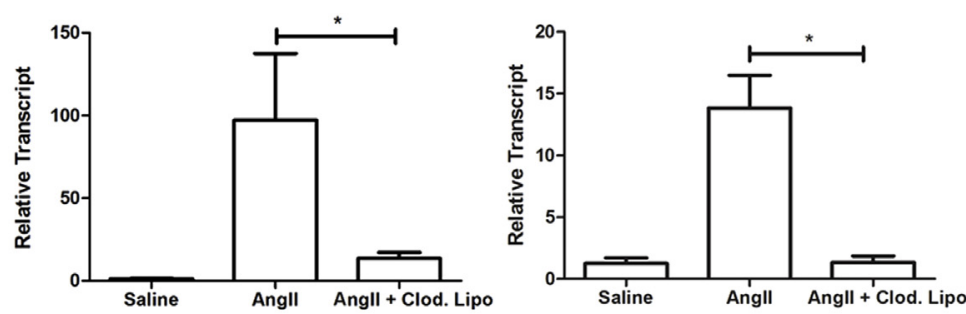


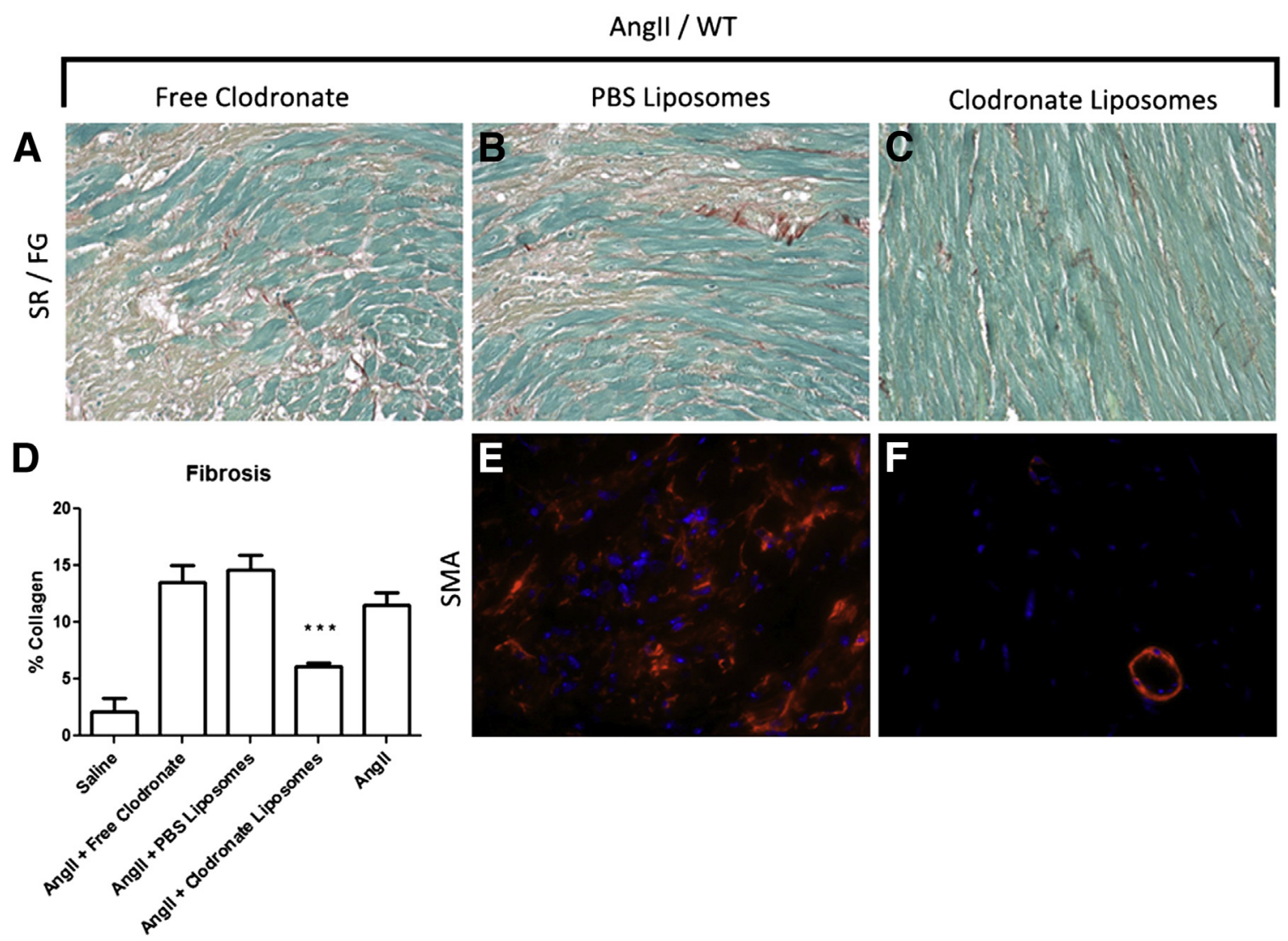

Figure 4 In the absence of the macrophage influx, myocardial fibrosis is significantly reduced. A-C: Representative images from myocardial sections stained with SR from mice that received AngII and clodronate solution (A), PBS liposomes (B), or clodronate liposomes (C) (collagen, red; cardiomyocytes, green). D: Quantification of SR/FG collagen content. E and F: Representative immunofluorescence showing large clusters of $\alpha$-SMA ${ }^{+}$fibroblasts in mice that received PBS liposomes (E) relative to those that received clodronate liposomes (F). Data are expressed as means \pm SEM. $n=3$ to 14 (D). ${ }^{* * *} P<0.001$. Original magnification: $\times 20($ A-C); $\times 40($ E and F). AngII, angiotensin II; FG, Fast Green; PBS, phosphate-buffered saline; SMA, smooth muscle cell actin; SR, Picosirius Red; WT, wild-type.

by monocytes as the mechanism for targeting these cells and supporting our previous work, ${ }^{15}$ suggesting that a significant number of myocardial infiltrating cells are derived from the circulation. The appearance of liposome-laden macrophages in the myocardium after AngII infusion supports the premise that these macrophages can be targeted in circulation by i.v. liposome administration.

\section{Monocyte Depletion Prevents Macrophage Accumulation in the AngII-Exposed Myocardium}

To confirm that monocytes could be depleted with cytotoxic clodronate liposomes, mice were intravenously administered liposomes that contained either PBS or clodronate, beginning 1 day before AngII infusion and each subsequent day for a total of four treatments. Nonliposome mice infused with AngII served as controls. After four treatments with liposomes, blood samples were characterized for monocyte populations by using flow cytometry and taken for smears and stained for cell morphology by using Giemsa. Flow cytometry supported a reduction of approximately $50 \%$ in $\mathrm{F} 4 / 80^{+} \mathrm{CD} 11 \mathrm{~b}^{+}$cells in mice that received clodronate liposomes (Figure 3C) relative to PBS liposomes (Figure 3A), which was quantified with blood smears. The ablation of monocytes with clodronate liposomes was consistent with the Ly6C expression profile of new monocytes (Ly6C $\mathrm{C}^{\text {high }}$ )

Figure 3 Monocyte depletion significantly decreases the macrophage influx after AngII infusion. A and B: Representative gating of a double-positive population (boxed area) in an F4/80 $\times$ CD11b plot from an animal that received PBS liposomes (A) and its Ly6C expression (B). C and D: Representative gating of the same double-positive population (boxed area) from an animal that received clodronate liposomes (C) and its Ly6C expression (D). E: Percentages of lymphocytes, neutrophils, monocytes, eosinophils, and basophils according to blood film nuclei counts. F: Magnified percentages of monocyte percentages from blood film nuclei counts. G-I: Representative images from myocardial H\&E sections from mice that received AngII and clodronate solution (G), PBS liposomes $(\mathbf{H})$, or clodronate liposomes (I). J: Quantification of area of heart affected by mononuclear infiltrate. $\mathbf{K}$ and $\mathbf{L}$ : Immunofluorescence confirms that mice that received PBS liposomes (K) have myocardial CD11 b $\mathrm{b}^{+}$populations that are absent in mice that received clodronate liposomes (L). $\mathbf{M}$ and $\mathbf{N}: \mathrm{F} 4 / 80^{+}$ cells are also observed in mice that received PBS liposomes (M) but are not apparent in mice that received clodronate liposomes (N). $\mathbf{0}-\mathbf{Q}$ : Myocardial transcript levels of TGF- $\beta(\mathbf{0})$, collagen $1 \alpha 1(\mathbf{P})$, and $\mathrm{CX} 3 \mathrm{CL} 1(\mathbf{Q})$ after AngII infusion with and without clodronate liposome. Data are expressed as means \pm SEM. $n=5(\mathbf{F}) ; n=3$ to $14(\mathbf{J}) ; n=4$ to $6(\mathbf{0}-\mathbf{Q}) .{ }^{*} P<0.05,{ }^{* *} P<0.001$. Original magnification: $\times 20(\mathbf{G}-\mathbf{I}) ; \times 40(\mathbf{K}-\mathbf{N})$. AngII, angiotensin II; APC, allophycocyanin; Clod, clodronate; CX3CL1, chemokine (C-X3-C motif) ligand 1; Lipo, liposome; H\&E, hematoxylin and eosin; PBS, phosphate-buffered saline; PE, phycoerythrin; PerCp-Cy5.5, peridinin chlorophyll protein complex cyanine 5.5; TGF- $\beta$, transforming growth factor $\beta$; WT, wild-type. 
entering circulation from the bone marrow (Figure 3D) relative to those differentiating toward nonclassical $\left(\right.$ Ly6C ${ }^{\text {low }}$ ) monocytes in mice that received PBS liposomes (Figure 3B). A blind observer (A.F.) assessed cells on blood smears for morphology and counted leukocyte subsets to a total of 350 cells per slide. Nonmonocyte populations were unaffected by the administration of either PBS or clodronate liposomes (Figure 3E). Control and PBS liposome mice exhibited $4.26 \% \pm 0.20 \%$ and $3.53 \% \pm 0.36 \%$ of monocytes of the total leukocyte population, respectively (Figure 3, E and F). The clodronate liposome group, however, demonstrated a significant reduction in monocytes, exhibiting $0.51 \% \pm 0.08 \%$ of leukocytes $(P<0.001)$ (Figure 3, E and F). This represented an $88.0 \%$ and $85.5 \%$ reduction in circulating monocytes relative to control and PBS liposome mice, respectively. Thus, consistent with previous reports, i.v. clodronate liposomes can effectively deplete circulating monocytes. ${ }^{26}$

\section{Clodronate Liposomes Reduce Monocyte Infiltration in the AngII-Exposed Myocardium}

Mice were infused with AngII and intravenously injected with PBS liposomes, clodronate liposomes, or clodronate solution and were sacrificed at 3 days. Myocardia from these mice were assessed for the percentage of myocardium affected by infiltrating cells with the use of a standardized grid as previously described. ${ }^{7,16,21-23}$ AngII infusion led to obvious areas of cellular infiltrate in the myocardium as indicated by an abundance of perivascular and interstitial mononuclear cells; the same pattern of mononuclear cells was minimal or absent in saline controls as previously described. ${ }^{15}$ Myocardia from mice that received AngII and either PBS liposomes or clodronate solution demonstrated similar patterns of mononuclear infiltrate relative to AngII alone (Figures 1A and 3, G and $\mathrm{H}$ ). Quantitatively, AngII-exposed hearts exhibited $10.00 \% \pm 1.34 \%$ of heart grids affected by infiltrating cells, a significant increase over saline controls $(0.37 \% \pm 0.37 \%$; $P<0.001$ ) (Figure 3J). Consistent with AngII infusion alone, mice that received AngII and either PBS liposomes or clodronate solution had $14.66 \% \pm 1.23 \%$ and $13.53 \% \pm 1.50 \%$ of the heart affected, respectively (Figure 3J). In contrast, all of the above controls mice that received AngII and clodronate liposomes showed little infiltration of cells in the myocardium (Figure 3I). Myocardia from mice that received AngII and clodronate liposomes exhibited just $3.28 \% \pm 0.26 \%$ in area of myocardium affected by infiltrating cells, a significant $77.6 \%$ reduction in infiltrating cells relative to AngII-infused mice approaching that of control mice $(P<0.001)$ (Figure $3 \mathrm{~J})$. Immunofluorescence for $\mathrm{CD} 11 \mathrm{~b}$ (Figure 3L) and F4/80 (Figure 3N) in myocardia from mice that received AngII and liposomes displayed little to no positivity, confirming our findings.

Consistent with AngII-infused mice, those that received PBS liposomes and AngII still showed populations of $\mathrm{CD} 1 \mathrm{~b}^{+}$(Figure $3 \mathrm{~K}$ ) and $\mathrm{F} 4 / 80^{+}$(Figure $3 \mathrm{M}$ ), indicative of macrophages. AngII led to significantly increased cardiac transcript levels of TGF- $\beta(4.706 \pm 0.3498)$ relative to mice that received saline $(1.774 \pm 0.9107 ; P<0.05)$ (Figure 3O). Surprisingly, clodronate liposomes in association with AngII further increased cardiac transcript levels of TGF- $\beta$ $(10.96 \pm 2.693)$ relative to mice that received AngII alone $(P<0.05)$ (Figure 3O). In contrast, collagen $1 \alpha 1$ $(13.62 \pm 3.612)$ and CX3CL1 $(1.335 \pm 0.5201)$ transcript levels were significantly reduced relative to mice that received AngII alone (97.16 \pm 40.31 and $13.84 \pm 2.649$, respectively; $P<0.05$ ) (Figure $3, \mathrm{P}$ and $\mathrm{Q}$ ), potentially supporting modulated nonclassical macrophage involvement.

\section{Reduction in Infiltrating Monocytes Is Associated with Reduced Myocardial Fibrosis}

After the analysis of infiltrating cells, representative myocardia from individual groups were stained for collagen by using the SR/FG stain as previously described. ${ }^{21,22}$ Stained myocardia were assessed for the percentage of collagen in whole myocardial sections. Large areas of collagen deposition were observed in mice that received AngII, as indicated by SR staining, and were significantly higher than saline control as previously described. ${ }^{15,21,22}$ Similar patterns of excess collagen deposition were observed in mice that received AngII and either PBS liposomes or clodronate solution (Figure 4, A and B, respectively). In contrast, mice that received AngII and clodronate liposomes had a significant reduction in collagen deposition compared with AngII mice; rather collagen was largely localized to vasculature, as in saline controls. Quantitatively, collagen comprised $11.50 \% \pm 1.12 \%$ of representative myocardial sections from mice that received AngII alone (Figure 4D). Consistent with infiltrating cell data, this represented a significant increase over basal collagen levels observed in saline controls $(2.11 \% \pm 1.25 \% ; P<0.001)$ (Figure 4D). Myocardia from mice that received AngII and either PBS liposomes or clodronate in solution demonstrated similar changes $(14.47 \% \pm 1.76 \%$ and $11.11 \% \pm 0.69 \%$, respectively), which were not significantly different from AngII alone (Figure 4D). In contrast, mice that received AngII and clodronate liposomes (Figure 4C) had a significant reduction $(3.28 \% \pm 0.26 \%)$ in myocardial collagen content; $71.5 \%$ reduction in collagen content relative to myocardia from mice that received AngII alone, corresponding to the significant reductions in infiltrating cells $(P<0.001)$ (Figure 4D). Mice that received AngII (and PBS liposomes) displayed clusters of $\alpha$-SMA ${ }^{+}$fibroblasts (Figure $4 \mathrm{E}$ ), an observation consistent with our previous findings in mice that received AngII alone. ${ }^{15}$ In support of the reduction in collagen content, mice infused with AngII that received clodronate liposomes did not have clusters of $\alpha-\mathrm{SMA}^{+}$fibroblasts, suggesting reduced fibroblast differentiation to activated myofibroblast phenotype and supporting reduced fibrosis (Figure $4 \mathrm{~F}$ ). Rather, $\alpha-\mathrm{SMA}^{+}$cells were primarily limited to vasculature as previously observed in historical controls and likely representative of smooth muscle cells that also can 
M $\theta$ Monoculture
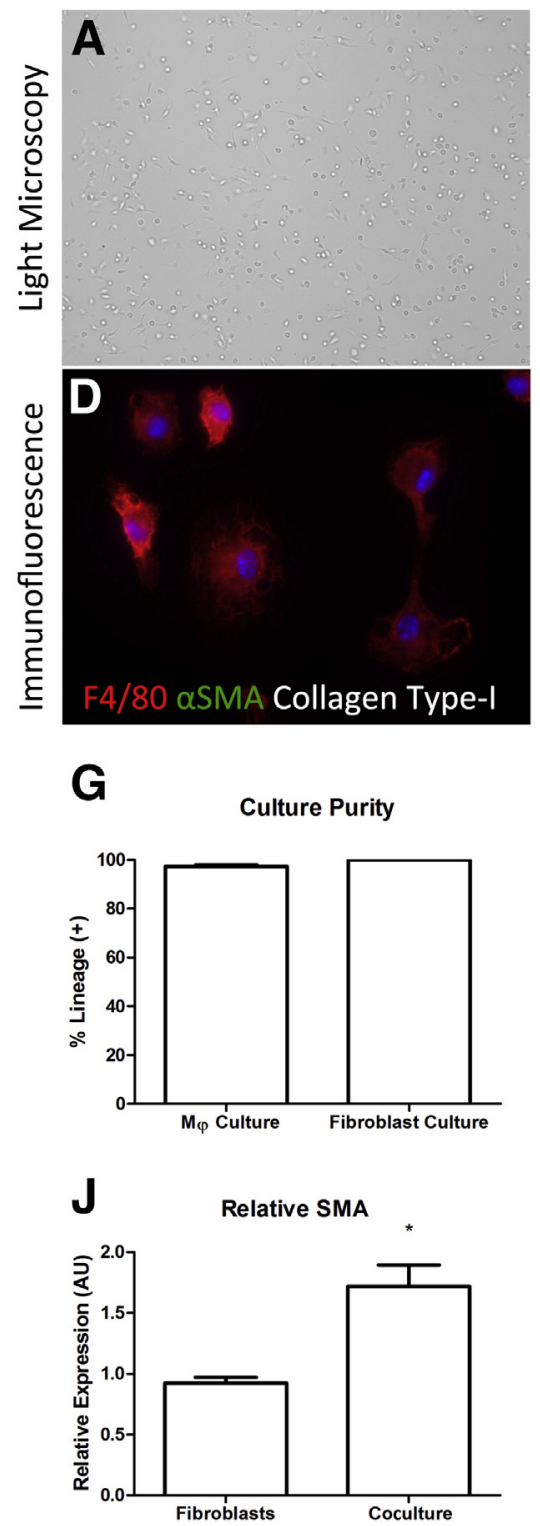

Fibroblast Monoculture
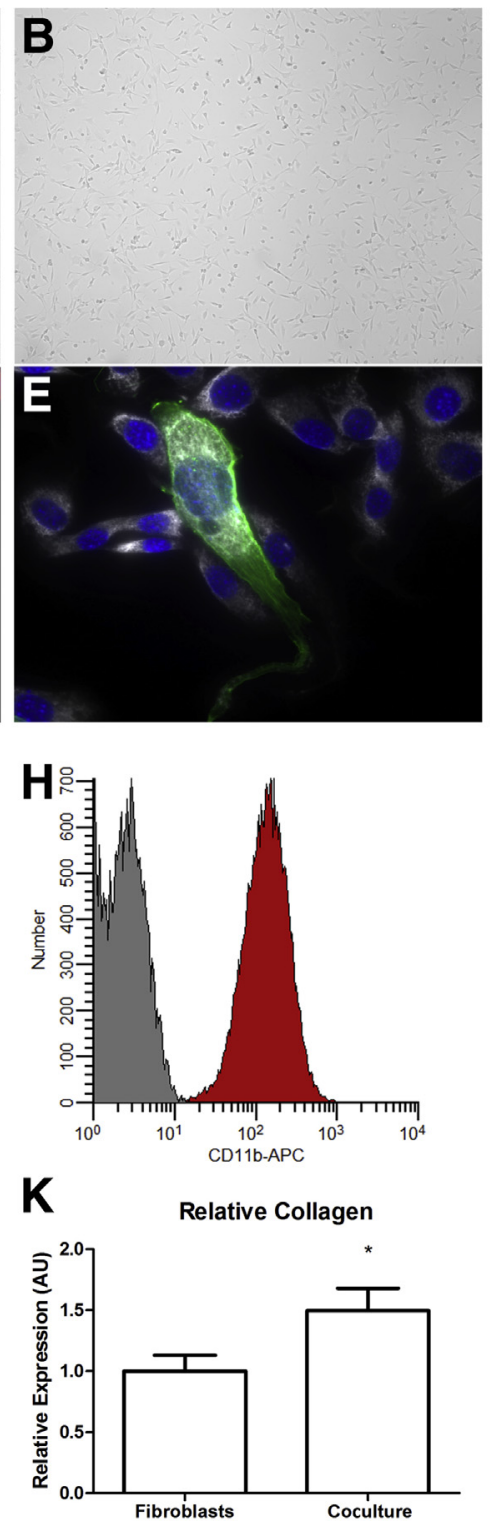

\section{Coculture}

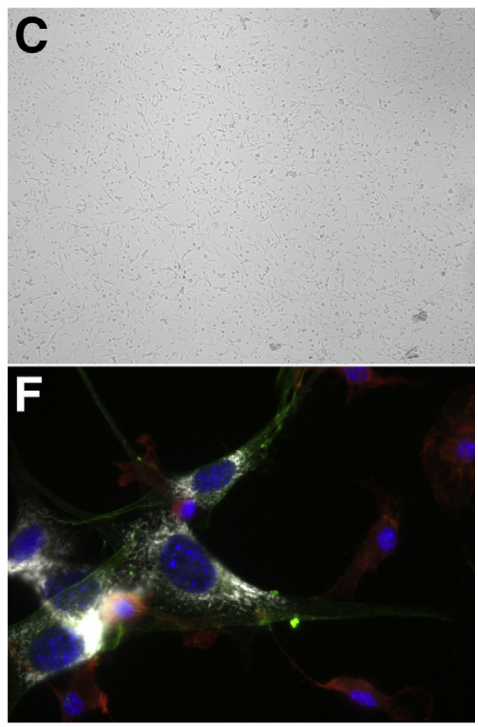

I

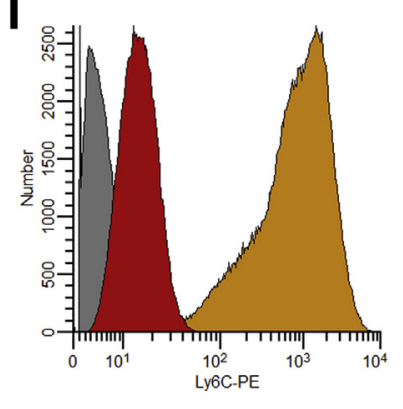

Figure 5 Nonclassical macrophages promote fibroblast activation and collagen production in vitro. BMDMs and 3 T3 fibroblasts were cultured in monoculture and coculture at equal ratio. Macrophage and fibroblast monocultures and coculture were characterized with light microscopy (A, B, and $\mathbf{C}$, respectively) and immunofluorescence for $F 4 / 80$ (red), collagen type-I (white), and $\alpha-S M A^{+}$(green) (D, E, and $\mathbf{F}$, respectively). G: Monoculture purity was quantified from immunofluorescence. $\mathbf{H}$ and I: BMDMs were further characterized for CD11b (H) and Ly6C (I) by using flow cytometry. J and K: Quantification of coculture expression demonstrates that nonclassical macrophages promote both fibroblast activation as indicated by $\alpha-\mathrm{SMA}^{+}(\mathbf{J})$ and collagen production $(\mathbf{K})$ relative to monocultures. Data are expressed as means \pm SEM. $n=6(\mathbf{G}) ; n=4$ to $6(\mathbf{J}$ and $\mathbf{K}) .{ }^{*} P<0.05$. Original magnification: $\times 10(\mathbf{A}-\mathbf{C}) ; \times 63(\mathbf{D}-\mathbf{F})$. APC, allophycocyanin; AU, arbitrary unit; BMDM, bone marrow-derived macrophage; $\mathrm{M} \theta$ or M $\varphi$, macrophage; macrophage; PE, phycoerythrin; $\alpha$-SMA, $\alpha$-smooth muscle cell actin.

express $\alpha$-SMA (data not shown). Thus, the reduction in macrophage observed with clodronate liposome treatment is associated with significant reductions in myocardial collagen, potentially because of reduced fibroblast proliferation and/or activation into myofibroblasts.

\section{Macrophages Promote Fibroblast $\alpha$-SMA Expression in Vitro}

Given the reduction in collagen and $\alpha$-SMA ${ }^{+}$cells in the myocardia of mice depleted of monocyte-derived macrophages, we evaluated whether macrophages could directly promote fibroblast activation. To characterize macrophage and fibroblast interactions, we set up a system of monocultures and juxtacrine cocultures.

After 2 days of culture, macrophages (Figure 5A), fibroblasts (Figure 5B), and cocultures (Figure 5C) were photographed under light microscopy and harvested for immunofluorescence. BMDMs were characterized for purity by using immunofluorescence for F4/80 (Figure 5D). The 3T3 fibroblasts were characterized for purity and activation by the expression of collagen and $\alpha$-SMA, respectively 

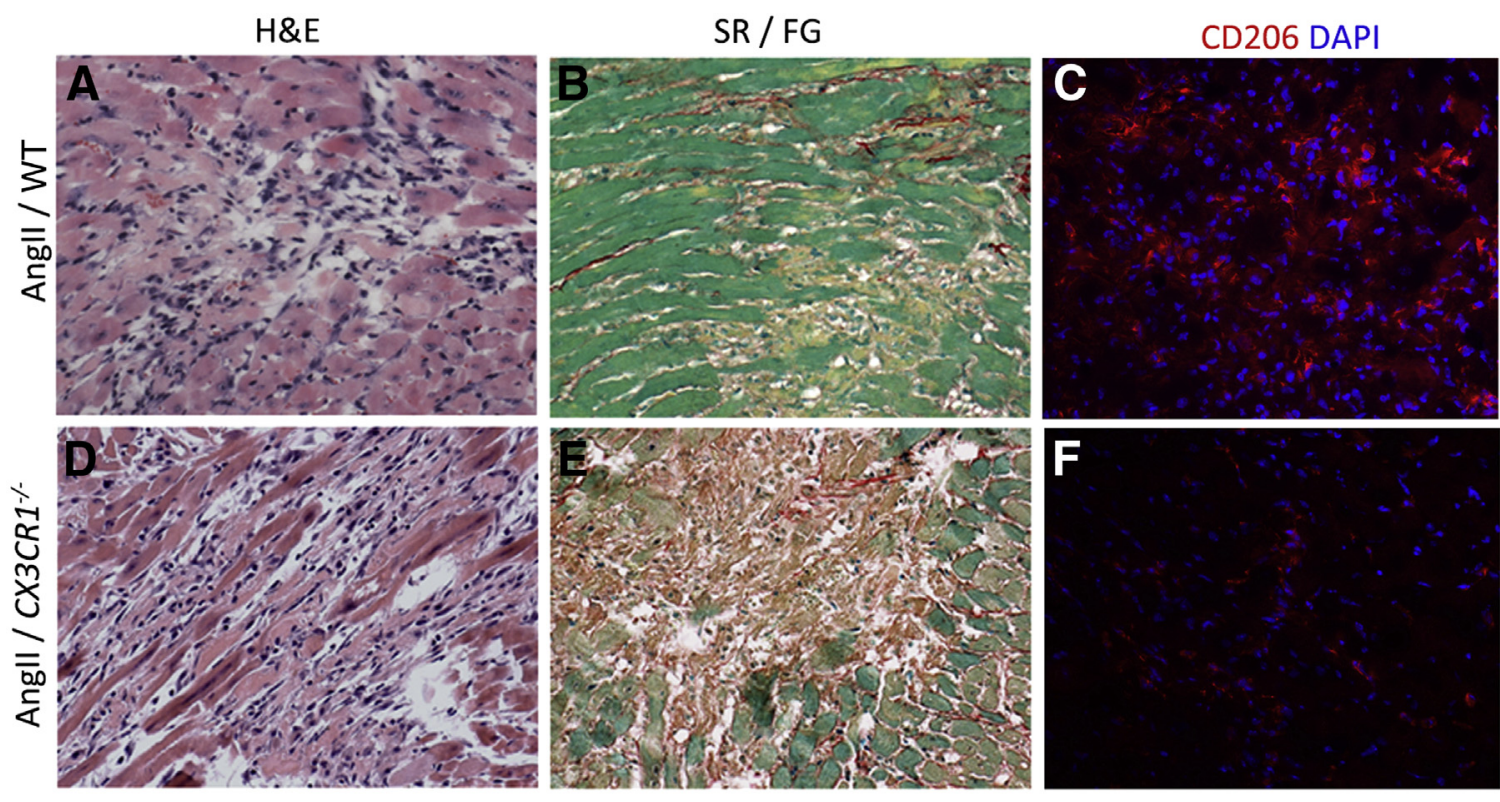

G

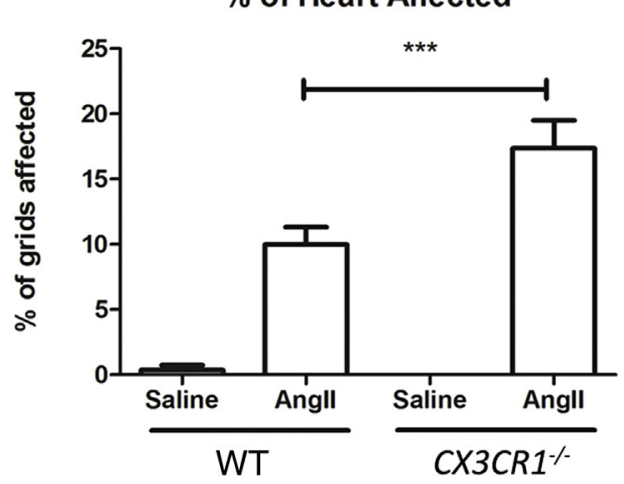

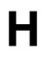

H

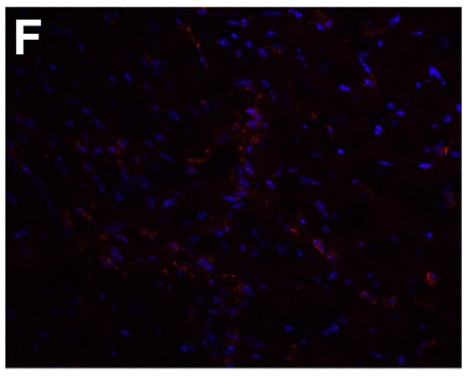

Fibrosis

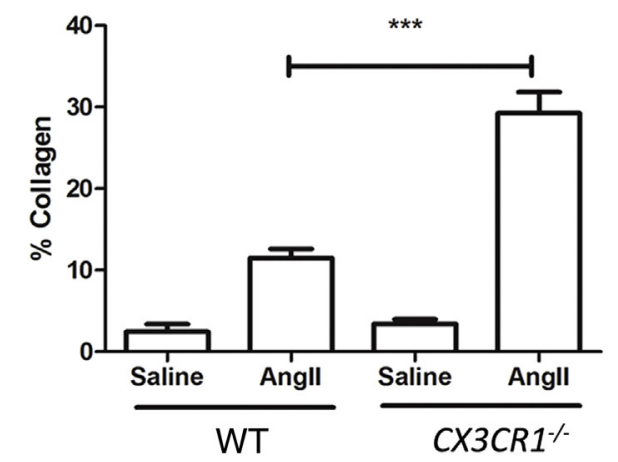

Figure 6 Nonclassical macrophage inhibition exacerbates myocardial infiltration and fibrosis after AngII infusion. Representative heart sections from AngII-infused WT $(\mathbf{A}-\mathbf{C})$ and $C \times 3 \mathrm{cr}^{-/-}$mice $(\mathbf{D}-\mathbf{F})$; mice were stained with $\mathrm{H \& E}, \mathrm{SR} / \mathrm{FG}$, and CD206, respectively. $\mathbf{G}$ and $\mathbf{H}$ : Semiquantification of infiltrate $(\mathbf{G})$ and fibrosis $(\mathbf{H})$. Saline-treated $\mathrm{C}_{3} 3 \mathrm{Cr}^{-1-}$ and WT mice were unremarkable. Data are expressed as means $\pm \mathrm{SEM} .{ }^{* * *} P<0.001$. Original magnification: $\times 20$ (A-F). AngII, angiotensin II; FG, Fast Green; H\&E, hematoxylin and eosin; SR, Picosirius Red; WT, wild-type.

(Figure 5E). The purity of macrophages and fibroblasts was $97.3 \% \pm 0.77 \%$ and $100.0 \% \pm 0.0 \%$, respectively $(P<0.001)$ (Figure $5 \mathrm{G})$. The $\mathrm{CD}_{11} \mathrm{~b}^{+}$cultured macrophages (Figure $5 \mathrm{H}$ ) were also found to exclusively express the Ly6 $\mathrm{C}^{\text {low }}$ nonclassical phenotype (Figure 5I). We demonstrated increased $\alpha$ $\mathrm{SMA}^{+}$fibroblast differentiation in the coculture compared with the monoculture, as seen in the representative fields of view (Figure 5, E and F). The expression of collagen and $\alpha$ SMA was quantified relative to cell number by standardizing to the fluorescence intensity of nuclei per well of cultured cells. A significant difference in collagen and $\alpha$-SMA expression was observed in cocultures relative to fibroblast monocultures $(P<0.05)$ (Figure 5, J and $\mathrm{K}$ ). It follows that macrophages, specifically of the nonclassical phenotype, are sufficient to promote fibroblast activation and collagen production by promoting myofibroblast differentiation.

Together, most Ly6C ${ }^{\text {low }}$ macrophage phenotype observed in the myocardium after AngII infusion, the reduction in
CX3CL1 myocardial transcript after monocyte depletion, and the coculture data suggest that nonclassical macrophages may have an important role in the development of AngIImediated myocardial fibrosis. To further evaluate the role of nonclassical monocyte recruitment in this process, we administered AngII to $\mathrm{CX} 3 \mathrm{CR} 1^{-/-}$mice and characterized the effects on cellular infiltrate and myocardial fibrosis.

\section{CX3CR1-Dependent Nonclassical Monocyte Recruitment} Is Protective in AngII-Mediated Myocardial Fibrosis

The absence of $C x 3 c r l$ on the surface of circulating monocytes did not result in any reduction in cellular infiltration as predicted (Figure 6D) compared with the cellular infiltrate in AngII-exposed WT myocardium (Figure 6A). In contrast, myocardial changes appeared to be exacerbated in the AngIIinfused $\mathrm{Cx} 3 \mathrm{crl}^{-1-}$ mice (Figure 6E) relative to AngII/WT (Figure 6B). In accordance with the Cx3crl-dependent 
$\mathrm{F} 4 / 80^{+}$gate (CD11b vs. Ly6C)
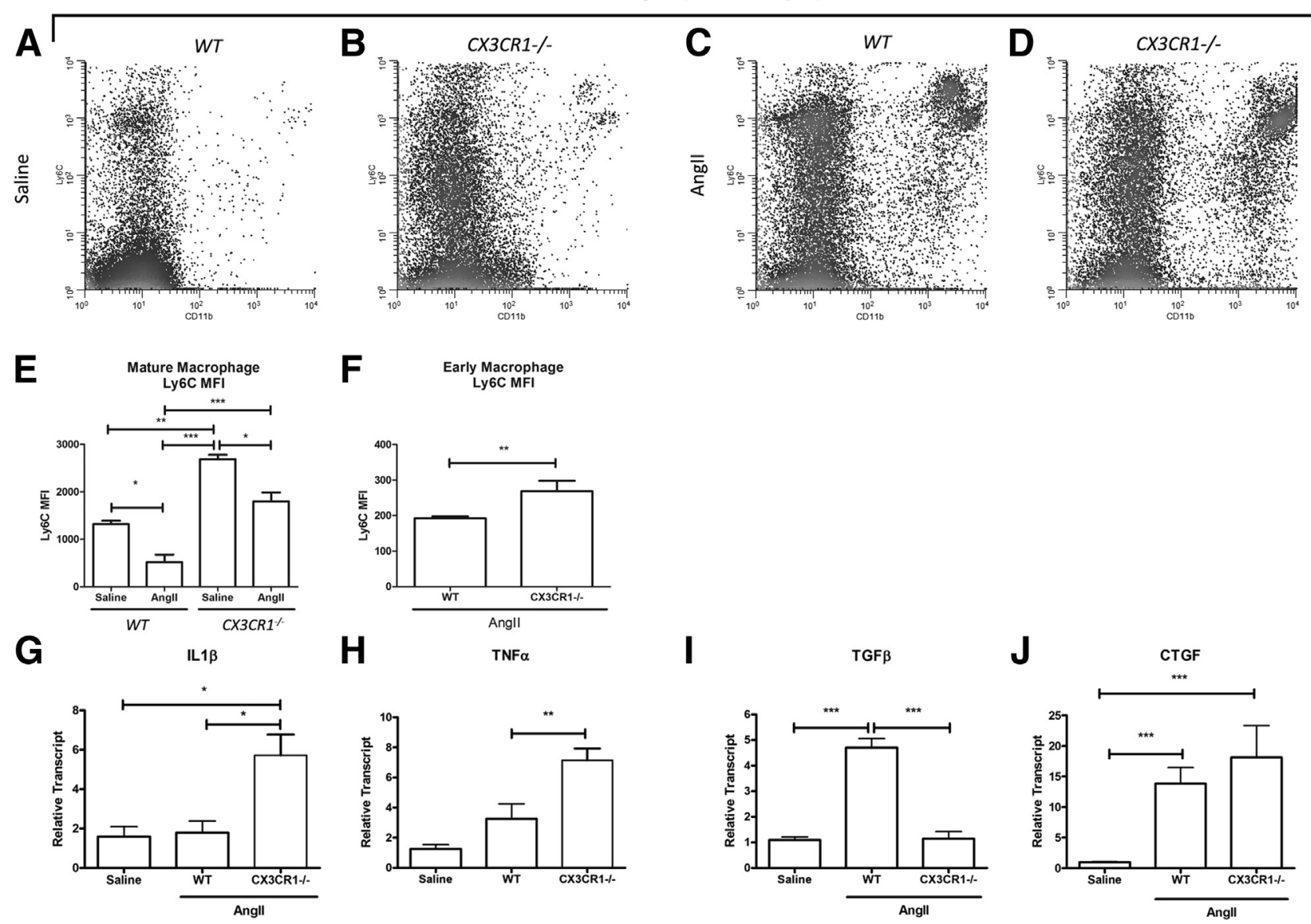

Figure 7 Resident and infiltrating macrophages in $\mathrm{C}_{3} 3 \mathrm{Cr}^{-/-}$myocardia favor a proinflammatory phenotype and environment. Representative flow cytometry for F4/80, CD11b, and Ly6C in saline-infused WT (A) and $\mathrm{Cx}_{3} \mathrm{Cr}^{-1^{-1}}$ (B) myocardia and AngII-exposed WT (C) and Cx3cr1 ${ }^{-/-}$(D) myocardia. Resident or mature macrophages are on the left side of dot plots, and infiltrating or early macrophages are on the right side of dot plots. MFI of Ly6C in resident (E) and infiltrating (F) cardiac macrophages from both $\mathrm{C}_{3} \mathrm{Cr}^{-1-}$ and WT mice. Infiltrating macrophages could only be characterized in mice that received AngII. Myocardial transcript levels of IL-1 $\beta(\mathbf{G})$, TNF- $\alpha(\mathbf{H})$, TGF- $\beta(\mathbf{I})$, and CTGF (J). Data are expressed as means \pm SEM. ${ }^{*} P<0.05$, ${ }^{* *} P<0.01$, and ${ }^{* * *} P<0.001$. AngII, angiotensin II; CTGF, connective tissue growth factor; MFI, mean fluorescent index; TGF- $\beta$, transforming growth factor- $\beta$; TNF- $\alpha$, tumor necrosis factor $\alpha$; WT, wild-type.

nonclassical monocyte recruitment, there was a marked reduction in myocardial CD206 ${ }^{+}$(a marker of M2 nonclassical macrophages) cells in the AngII-infused $\mathrm{Cx} 3 \mathrm{crl}^{-/-}$ mice (Figure 6F) compared with AngII WT counterparts assessed by immunocytochemistry (Figure 6C). Cellular infiltrate was significantly increased in AngII-infused $\mathrm{Cx}_{3} \mathrm{crl}^{-1-}$ mice $(17.37 \% \pm 2.13 \%$ of heart grids affected $)$ relative to WT controls $(10.00 \% \pm 1.34 \%$ of heart grid affect) (Figure 6G). Furthermore, the increase in myocardial infiltrate in $\mathrm{Cx} 3 \mathrm{crl}^{-1-}$ mice was associated with a significant 2.54 -fold increase in collagen deposition $(29.26 \% \pm 2.58 \%)$ relative to WT mice $(11.50 \% \pm 1.12 \%)$ (Figure $6 \mathrm{H})$. Saline controls from both $\mathrm{C} x 3 \mathrm{crl}^{-/-}$and WT mice were unremarkable (Figure $6 \mathrm{H}$ ). On the basis of the apparent reduction in myocardial CD206 expression in $\mathrm{Cx}_{3} \mathrm{crl}^{-1-}$ mice relative to WT mice, we suspected that there was a shift toward a classical or intermediate phenotype and that nonclassical phenotype may provide a yet-to-be-described protective role.

\section{Total Macrophages in $\mathrm{C}_{3} \mathrm{Cr}^{-/-}$AngII-Exposed Hearts Display Higher Ly6C Expression}

After AngII infusion, both WT and $C \times 3 \mathrm{CrI}^{-/-}$total cardiac macrophage populations (resident + infiltrating) shifted toward lower Ly6C expression relative to saline controls (Figure 7, C and $\mathrm{D})$. When quantified, it was found that the reduction in cardiac Ly6C expression between saline- and AngII-infused mice was significantly reduced $(P<0.05)$ (Figure $7 F)$. In contrast total cardiac macrophages in AngII/ $\mathrm{C} \times 3 \mathrm{crl}^{-/-}$mice continued to demonstrate a significant shift toward higher Ly6C expression relative to AngII/WT mice, favoring a proinflammatory phenotype $(P<0.001)$ (Figure 7 F).

\section{Infiltrating Macrophages in $\mathrm{C} \times 3 \mathrm{Cr}^{-/-}$AngII-Exposed Hearts Display Higher Ly6C Expression}

Despite the presence of multiple dense, Ly6C $\mathrm{C}^{\text {high }}$, early macrophage populations in the AngII-infused WT hearts, 
Table 1 Physiologic and Morphologic Indicators

\begin{tabular}{|c|c|c|c|c|c|}
\hline Strain & $1^{\circ}$ Treatment & $2^{\circ}$ Treatment & $\mathrm{HW} / \mathrm{BW}, \mathrm{mg} / \mathrm{g}$ & $\Delta B W, g$ & $\mathrm{MABP}, \mathrm{mmHg}$ \\
\hline \multirow[t]{5}{*}{ WT } & Saline & No liposomes & $5.19 \pm 0.18$ & $0.23 \pm 0.04$ & $101.3 \pm 4.22$ \\
\hline & AngII & No liposomes & $6.15 \pm 0.15^{*}$ & $-2.30 \pm 0.21^{*}$ & $130.6 \pm 5.32^{* *}$ \\
\hline & & Clodronate liposomes & $6.23 \pm 0.16^{*}$ & $-2.79 \pm 0.22^{*}$ & $129.8 \pm 2.88^{* *}$ \\
\hline & & PBS liposomes & $6.38 \pm 0.17^{\star}$ & $-2.37 \pm 0.21^{*}$ & $123.3 \pm 5.21^{* *}$ \\
\hline & & Free clodronate & $6.33 \pm 0.26^{*}$ & $-2.85 \pm 0.48^{*}$ & $124.4 \pm 6.08^{* *}$ \\
\hline \multirow[t]{2}{*}{$\mathrm{C} \times 3 \mathrm{Cr1}^{-/-}$} & Saline & No liposomes & $4.93 \pm 0.28$ & $0.29 \pm 0.07$ & $103.5 \pm 2.41$ \\
\hline & AngII & No liposomes & $7.48 \pm 0.52^{\dagger}$ & $-3.64 \pm 0.37^{\dagger}$ & $125.7 \pm 4.57^{* *}$ \\
\hline
\end{tabular}

Data are expressed as means \pm SEM.

${ }^{*} P<0.001,{ }^{*} P<0.01$ versus saline/WT.

${ }^{\dagger} P<0.001$ versus AngII/WT.

AngII, angiotensin II; HW/BW, heart weight/body weight; MABP, mean arterial blood pressure; WT, wild-type; $\Delta$ BW, change in body weight.

the early macrophage populations in $\mathrm{Cx} 3 \mathrm{crl}^{-/-}$mice demonstrated a significant increase in mean fluorescent index of Ly6C $(P<0.01)$ (Figure $7 \mathrm{~F})$. This 1.4-fold increase relative to AngII-infused WT mice suggests a shift toward the proinflammatory, classical macrophage phenotype, which could further exacerbate an overall proinflammatory environment set by the resident population.

\section{C $\times 3 \mathrm{Cr}^{-1-}$ AngII-Exposed Mice Have a Proinflammatory Myocardial Environment}

In support of the hypothesis that phenotypic proinflammatory macrophages favor an inflammatory cardiac environment, myocardial transcript levels of the proinflammatory cytokines IL-1 $\beta$ and TNF- $\alpha$ were significantly elevated in $\mathrm{C} \times 3 \mathrm{crl}^{-/-}$relative to WT AngII-infused mice $(P<0.05$ and $P<0.01$, respectively) (Figure $7, \mathrm{G}$ and $\mathrm{H}$ ). In addition, AngII-infused $\mathrm{Cx} 3 \mathrm{CrI}^{-/-}$mice displayed a significant reduction in myocardial transcript levels of the antiinflammatory cytokine TGF- $\beta(P<0.001)$ (Figure $7 \mathrm{I}$ ), while maintaining myocardial transcript levels of the profibrotic CTGF (Figure 7J).

\section{Resident Macrophages in $\mathrm{C} \times 3 \mathrm{Cr}^{-/-}$Control Hearts Display Higher Ly6C Expression}

To compare macrophage phenotypes in the hearts of control WT and control $\mathrm{Cx} 3 \mathrm{crl}^{-1-}$ mice, we isolated nonmyocytes after 3 days of saline infusion. Cells were labeled for F4/80,
CD11b, and Ly6C as described in Materials and Methods. In both saline-infused WT (Figure 7A) and $\mathrm{Cx3} \mathrm{crl}^{-/-}$ (Figure 7B) control hearts, there were readily identifiable populations of $\mathrm{F} 4 / 80^{+} \mathrm{CD} 11 \mathrm{~b}^{\text {low }}$ cells observed in the left half of the dot plots, indicative of resident cardiac macrophages. These resident cardiac macrophages displayed a spectrum of Ly6C expression ( $y$ axis); however, the resident cardiac macrophages in the $\mathrm{Cx} 3 \mathrm{crl}^{-/-}$hearts appeared to favor a Ly6C ${ }^{\text {mid }}$ phenotype, more consistent with the classical or intermediate phenotype. Quantification of Ly6C expression by mean fluorescence index confirmed that, even at baseline levels, Ly6C expression in $\mathrm{Cx}_{3} \mathrm{crl}^{-/-}$cardiac resident macrophages significantly exceeded baseline levels in WT controls $(P<0.01)$ (Figure $7 \mathrm{~F})$. Thus, if increased Ly6C expression indicates a proinflammatory macrophage phenotype, then $\mathrm{Cx} 3 \mathrm{crl}^{-/-}$mice may have a predisposition to cardiac inflammation or, conversely, reduced protection to inflammation which could explain their higher susceptibility to AngII.

\section{Differences in Cellular Infiltrate and Collagen Deposition Independent of Physiologic Indicators}

AngII infusion led to a significant increase in mean arterial blood pressure relative to saline controls (130.6 \pm 5.32 $\mathrm{mmHg})$ compared with controls $(101.3 \pm 4.22 \mathrm{mmHg}$; $P<0.001$ ) (Table 1). WT mice that received PBS liposomes, clodronate liposomes, or clodronate solution and $\mathrm{Cx} 3 \mathrm{crl}^{-/-}$ mice responded to AngII with similar increases in mean

Table 2 Echocardiography Measurements

\begin{tabular}{|c|c|c|c|c|c|c|}
\hline Strain & $1^{\circ}$ Treatment & $2^{\circ}$ Treatment & LVPWs, $\mathrm{mm}$ & LWPWd, mm & IVSWs, $\mathrm{mm}$ & IVSWd, $\mathrm{mm}$ \\
\hline \multirow[t]{2}{*}{$\overline{W T}$} & Saline & No liposomes & $1.23 \pm 0.10$ & $0.92 \pm 0.08$ & $1.36 \pm 0.17$ & $1.08 \pm 0.14$ \\
\hline & AngII & No liposomes & $1.80 \pm 0.22^{*}$ & $1.20 \pm 0.13^{*}$ & $1.73 \pm 0.14^{*}$ & $1.51 \pm 0.14^{*}$ \\
\hline \multirow[t]{2}{*}{$\mathrm{C} \times 3 \mathrm{Cr}^{-/-}$} & Saline & No liposomes & $1.20 \pm 0.08$ & $0.85 \pm 0.09$ & $0.98 \pm 0.02$ & $0.81 \pm 0.03$ \\
\hline & AngII & No liposomes & $1.65 \pm 0.08^{*}$ & $1.24 \pm 0.06^{*}$ & $1.29 \pm 0.10^{\dagger}$ & $1.09 \pm 0.09^{\dagger}$ \\
\hline
\end{tabular}

Data are expressed as means \pm SEM.

$* P<0.05$ versus saline/WT.

${ }^{\dagger} P<0.05$ versus AngII/WT.

AngII, angiotensin II; IVSWd, intraventricular septal wall thickness in diastole; IVSWs, intraventricular septal wall thickness in systole; LVPWd, left ventricular posterior wall thickness in diastole; LVPWs, left ventricular posterior wall thickness in systole; WT, wild-type. 
arterial blood pressure $(123.3 \pm 5.21 \mathrm{mmHg}, 129.8 \pm 2.88$ $\mathrm{mmHg}, 124.4 \pm 6.01 \mathrm{mmHg}$, and $125.7 \pm 4.57 \mathrm{mmHg}$, respectively) (Table 1). Mean arterial blood pressure did not significantly differ between experimental groups that received AngII with or without liposomes or clodronate solution. Thus, the overall observations in this study appear to be blood pressure independent. Similar to blood pressure, mice that received AngII exhibited significant weight loss $(-2.30 \pm 0.21 \mathrm{~g})$ and myocardial hypertrophy $(6.15 \pm 0.15$ $\mathrm{mg} / \mathrm{g})$ relative to saline controls $(+0.23 \pm 0.04 \mathrm{~g}$ versus $5.19 \pm 0.18 \mathrm{mg} / \mathrm{g}$ ) (Table 1). Weight loss and hypertrophy were comparable between experimental groups that received AngII independent of liposomes or clodronate solution. AngII-infused $\mathrm{Cx} 3 \mathrm{crl}^{-/-}$mice also exhibited significantly increased hypertrophy $(7.48 \pm 0.52 \mathrm{mg} / \mathrm{g})$ relative to AngIIinfused WT mice $(P<0.01)$ (Table 1). Finally, transthoracic echocardiography suggested that both AngII/WT and AngII/ $\mathrm{C} \times 3 \mathrm{CrI}^{-/-}$mice exhibited significantly increased left ventricular posterior wall and intraventricular septal wall thicknesses during systole and diastole relative to their respective controls, supporting the findings of hypertrophy $(P<0.05)$ (Table 2).

\section{Discussion}

Macrophages are experiencing a renaissance in research, largely in part to the recent discoveries of their phenotypic and functional heterogeneity. ${ }^{31-33}$ Macrophage function is now known to span from proinflammatory to antiinflammatory to proangiogenic to profibrotic, which together serve an essential role in tissue healing, particularly in the context of the myocardium. ${ }^{31,33-39}$ It is suggested that the role of macrophages in ischemic injury is characterized by a biphasic response, in which early classical macrophages drive the inflammatory phase and nonclassical macrophages resolve the inflammation and promote remodeling. ${ }^{35,38}$ The role of macrophages in nonischemic models such as hypertension secondary to AngII infusion is less clear. In the wellestablished AngII-dependent model of hypertension, we and others have demonstrated an absence of tissue necrosis and associated polymorphonuclear cell infiltrates, suggesting that this model may not require the mass influx of classical macrophages. ${ }^{7,15,16,21,23}$ We have also characterized that early myocardial infiltration in AngII-exposed hearts is largely unaffected in $C \mathrm{Cr} 2^{-/-}$mice, suggesting redundancy in chemokines or implicating $C x 3 c r l$-dependent nonclassical macrophage recruitment. ${ }^{21}$ Despite this body of evidence it remained to be demonstrated whether macrophages were mediating the cellular changes observed in the myocardium and the ECM remodeling after AngII infusion. Here, we characterized the role of infiltrating macrophages via liposomal clodronate depletion and then characterized the contribution of $\mathrm{Cx} 3 \mathrm{crl}$-dependent nonclassical recruitment by using a $\mathrm{Cx} 3 \mathrm{Crl}^{-/-}$mice. ${ }^{11,40-42}$

As previously shown, a dramatic influx of mononuclear cells precedes the development of myocardial fibrosis during
AngII infusion. ${ }^{5,15,16}$ Here, we characterized the infiltrating cells as predominantly macrophages with further characterization on the basis of Ly6C expression. We were able to demonstrate an overall trend toward $\mathrm{Ly} 6 \mathrm{C}^{\text {low }}$ expression and CD206 positivity, consistent with nonclassical macrophages as the predominant phenotype of the accumulating cells within the myocardium. ${ }^{35,37,38}$ A similar mass influx of nonclassical macrophages is observed in ischemic myocardial injury and in nonischemic models of lung, liver, and kidney injury, thus supporting our findings. ${ }^{3,12-14,43}$ Nonclassical macrophages are generally associated with the production of profibrotic factors and the fibrotic phase of healing. ${ }^{35,44}$ Given the presence of nonclassical macrophages in the AngII-exposed myocardium and their profibrotic role, it followed that the nonclassical macrophages would be the key candidate cells capable to mediate and promote the observed myocardial fibrosis.

In our initial approach, we used i.v. clodronate liposomes to successfully deplete most circulating monocytes, as previously described. ${ }^{26,45}$ Monocyte depletion resulted in a significant reduction of macrophages in the heart after AngII infusion, which also resulted in significant reduction in myocardial fibrosis. Furthermore, the reduction in infiltration and fibrosis was independent of blood pressure. Other compensatory mechanisms observed with AngII infusion such as hypertrophy were not affected by macrophage depletion or the reduction in myocardial fibrosis. As such, other structural compensatory mechanisms may be sufficient to overcome the increased stress on the myocardium without the need for ECM fibrotic stabilization. This is in stark contrast to ischemic myocardial injury that requires early classical macrophages to remove necrotic tissue to progress to neovascularization and normal remodeling. In the absence of this classical macrophage response, the myocardium becomes structurally unstable and is associated with increased mortality. ${ }^{35,46-48}$ Nonclassical macrophage infiltrate then ensues to sufficiently replace lost tissue with ECM deposition (replacement fibrosis), a hallmark of myocardial fibrosis. ${ }^{35,46}$ As shown in our model, there is a robust infiltration of Ly6C ${ }^{\text {high }}$ macrophages after AngII infusion, but the overwhelming phenotype in the heart is Ly6 $\mathrm{C}^{\text {low }}$, which corresponded to a nonclassical resident population. We have also identified the presence of a nonclassical resident macrophage population in the heart of control mice before AngII exposure, supporting recent findings by Pinto et $\mathrm{al}^{17}$ about a significant resident macrophage population being present in the myocardium. Furthermore, this resident population expresses $\mathrm{Cx} 3 \mathrm{crl}$ and exhibits nonclassical, anti-inflammatory characteristics, but its exact contribution to the infiltrating population is unclear and beyond the scope of the present study. ${ }^{17}$ We suspect that liposomal depletion prevented the accumulation of infiltrating macrophages, but it may have left the resident population intact. Our findings suggest that the infiltrating population, expressing a Ly6 $\mathrm{C}^{\text {high }}$ phenotype, may be promoting inflammation, which is then counterbalanced by the resident $\mathrm{Ly} 6 \mathrm{C}^{\mathrm{low}}$ population. 
Surprisingly, we observed a significant increase in cardiac transcript of TGF- $\beta$ after clodronate liposome treatment. We acknowledge that TGF- $\beta$ is commonly associated with fibrotic conditions; however, the pleiotropic growth factor is also shown to have potent anti-inflammatory effects in the myocardium. ${ }^{49}$ Specifically, TGF- $\beta$ has deactivating properties that can prevent macrophage-mediated proinflammatory injury. ${ }^{50}$ Moreover, no studies have examined the specific contribution of resident cardiac macrophages to TGF- $\beta$ signaling. Although we did not investigate the mechanism by which TGF- $\beta$ may reduce myocardial fibrosis in our model, future work could take into consideration macrophage deactivation, particularly in the context of downstream signaling. The pleotropic effects of TGF- $\beta$ may be underlying our observations from the macrophagefibroblast coculture. Evidence suggests that IL-6 mediates fibroblast activation and collagen production via TGF- $\beta$ pathways. ${ }^{5}$ Although the combination of IL- 6 and TGF- $\beta$ signaling may promote a fibrotic response, a reduction of inflammatory IL-6 signaling, as may be the case after the depletion of infiltrating monocytes, may elicit an alternative, anti-inflammatory response. Ideally, one would examine the interactions between different macrophage activation states with fibroblasts; however, this is a shortcoming of BMDMs, which inevitably acquire a Ly6C ${ }^{\text {low }}$ phenotype in the absence of exogenous signaling and suggests that our findings need to be interpreted with these caveats. Thus, in the absence of the infiltrating macrophages, the resident population may confer some protection for the myocardium from hypertensive or inflammatory changes normally seen after AngII exposure.

Despite our important findings that monocyte depletion leads to obvious benefits for myocardial cellular infiltration and remodeling, it would be of little therapeutic value because of the systemic immunosuppressive effects. However, eventual refinement in techniques by using liposomes may provide novel yet-to-be-described opportunities to target specific monocyte subsets or to influence their phenotype. ${ }^{51}$ As such, liposomes could be used to modulate monocyte phenotype and function toward the anti-inflammatory, nonclassical phenotypic to reduce remodeling in the myocardium while maintaining immunocompetence and myocardial stability. Harel-Adar et $\mathrm{al}^{51}$ provided one such method by which macrophage phagocytosis of phosphatidylserine-presenting liposomes promoted the $\mathrm{CD} 206^{+}$, anti-inflammatory macrophage phenotype. Shifting the macrophage phenotype toward Ly6C ${ }^{\text {low }} \mathrm{CD}^{206}{ }^{+}$, as observed in AngII/WT myocardia, was sufficient to down-regulate proinflammatory cytokines such as tumor necrosis factor $\alpha$ and to increase antiinflammatory mediators such as TGF- $\beta$. This approach to shifting macrophage phenotype is shown to protect the myocardium after ischemic injury but has yet to be explored in nonischemic models of myocardial fibrosis.

Given the above-mentioned findings, we aimed to specifically inhibit nonclassical macrophage recruitment during
AngII infusion by using a chemokine receptor knockout for Cx3 crl, the predominant chemokine receptor expressed by nonclassical circulating monocytes. Recent evidence in skin, liver, and kidney suggests that $C x 3 \mathrm{Crl}^{-/-}$mice may display reduced fibrosis; thus, Cx3xcrl-dependent macrophage recruitment could represent a therapeutic opportunity for fibrosis and remodeling. ${ }^{41,42,52,53}$ Contrasting reports in a liver model suggested that impaired nonclassical macrophage recruitment exacerbated inflammation and consequently remodeling. ${ }^{11}$ Consistent with the liver model, we have demonstrated that, by inhibiting the recruitment of nonclassical macrophages, there is a shift toward a proinflammatory macrophage phenotype and environment in the heart, which resulted in worse myocardial cellular changes and remodeling. These findings could also be interpreted to suggest that nonclassical macrophages play an important regulatory role and are protective in maintaining ECM homeostasis. Furthermore, recent studies on resident cardiac macrophages which used green fluorescent protein under the Cx3crl promoter support that our findings are specific to the macrophages, because fibroblasts, endothelial cells, and cardiomyocytes were all negative. ${ }^{17}$

One needs to be cautious of oversimplification when trying to understand the differing roles of macrophage subsets. Although the infiltrating macrophage populations are obviously important in cardiac injury and remodeling, one needs to take into consideration resident macrophages, which we were able to identify in this study, but their exact role remains largely unknown. Our findings are consistent with the resident macrophage population, described by Pinto et al, ${ }^{17}$ based on their expression of $\mathrm{Cx} 3 \mathrm{crl}$, and an antiinflammatory phenotype. We have demonstrated for the first time that the resident macrophage population can shift phenotype in the absence of $C x 3 \mathrm{crl}$ signaling, something yet to be reported and perhaps important to our observations in which $C x 3 \mathrm{crl}^{-1-}$ mice had a shift in resident macrophages toward Ly6 $\mathrm{C}^{\text {high }}$, potentially making them more susceptible to AngII exposure. Although recent findings indicate that cardiac macrophages are largely replenished from selfrenewal, one cannot rule out that their initial recruitment is Cx3crl dependent. ${ }^{29,54}$ One also cannot rule out that $C x 3 c r l$ may be involved in macrophage survival, as suggested by Landsman et al. ${ }^{55}$ Our observations may depend on a resident cardiac macrophage population that would be unaffected by i.v. liposomal clodronate but modulated in the $\mathrm{Cx} 3 \mathrm{crl}^{-/-}$ mice. Because the resident cardiac macrophage population can be affected phenotypically and perhaps functionally by either modulated turnover or survival, they may still represent a valuable therapeutic target.

In summary, monocyte-derived macrophages are the primary infiltrating cell in the early development of AngIIdependent myocardial fibrosis. For the first time, we have demonstrated that depleting circulating monocytes prevents the accumulation of myocardial macrophages in nonischemic injury. In the absence of the macrophage influx, there is a marked reduction in cells expressing an activated 
fibroblast phenotype and a reduction in collagen deposition. Notably, we have demonstrated that the resident macrophage population in the heart can shift its phenotype and may be an important modulator of myocardial healing. Together, infiltrating and resident macrophages may be potential therapeutic targets in the development of nonischemic myocardial fibrosis. Future studies should examine whether modulating the phenotype of cardiac macrophages, rather than depleting all circulating monocytes, can lead to clinically important reductions in myocardial fibrosis while maintaining immune competence.

\section{Acknowledgment}

We thank Tanya Myers for technical support.

\section{References}

1. Leask A: Signaling in fibrosis: targeting the TGF beta, endothelin-1 and CCN2 axis in scleroderma. Front Biosci (Elite Ed) 2009, 1:115-122

2. Go AS, Mozaffarian D, Roger VL, Benjamin EJ, Berry JD, Blaha MJ, et al: Heart disease and stroke statistics-2014 update: a report from the American Heart Association. Circulation 2013, 129: e28-e292

3. Schulze PC, Lee RT: Macrophage-mediated cardiac fibrosis. Circ Res 2004, 95:552-553

4. Wang L, Li YL, Zhang CC, Cui W, Wang X, Xia Y, Du J, Li HH: Inhibition of Toll-like receptor 2 reduces cardiac fibrosis by attenuating macrophage-mediated inflammation. Cardiovasc Res 2014, 101: 383-392

5. Ma F, Li Y, Jia L, Han Y, Cheng J, Li H, Qi Y, Du J: Macrophagestimulated cardiac fibroblast production of IL-6 is essential for TGF $\beta /$ Smad activation and cardiac fibrosis induced by angiotensin II. PLoS One 2012, 7:e35144

6. Shen JZ, Morgan J, Tesch GH, Fuller PJ, Young MJ: CCL2dependent macrophage recruitment is critical for mineralocorticoid receptor-mediated cardiac fibrosis, inflammation, and blood pressure responses in male mice. Endocrinology 2014, 155:1057-1066

7. Sopel MJ, Rosin NL, Falkenham AG, Bezuhly M, Esmon CT, Lee TD, Liwski RS, Legare JF: Treatment with activated protein C $(\mathrm{aPC})$ is protective during the development of myocardial fibrosis: an angiotensin II infusion model in mice. PLoS One 2012, 7:e45663

8. Kania G, Blyszczuk P, Eriksson U: Mechanisms of cardiac fibrosis in inflammatory heart disease. Trends Cardiovasc Med 2009, 19:247-252

9. Shi C, Pamer EG: Monocyte recruitment during infection and inflammation. Nat Rev Immunol 2011, 11:762-774

10. Lin HH, Faunce DE, Stacey M, Terajewicz A, Nakamura T, ZhangHoover J, Kerley M, Mucenski ML, Gordon S, Stein-Streilein J: The macrophage F4/80 receptor is required for the induction of antigenspecific efferent regulatory $\mathrm{T}$ cells in peripheral tolerance. J Exp Med 2005, 201:1615-1625

11. Aoyama T, Inokuchi S, Brenner DA, Seki E: CX3CL1-CX3CR1 interaction prevents carbon tetrachloride-induced liver inflammation and fibrosis in mice. Hepatology 2010, 52:1390-1400

12. Heymann F, Trautwein C, Tacke F: Monocytes and macrophages as cellular targets in liver fibrosis. Inflamm Allergy Drug Targets 2009, 8:307-318

13. Vernon MA, Mylonas KJ, Hughes J: Macrophages and renal fibrosis. Semin Nephrol 2010, 30:302-317

14. Pechkovsky DV, Prasse A, Kollert F, Engel KM, Dentler J, Luttmann W, Friedrich K, Müller-Quernheim J, Zissel G: Alternatively activated alveolar macrophages in pulmonary fibrosis-mediator production and intracellular signal transduction. Clin Immunol 2010, 137:89-101

15. Sopel MJ, Rosin NL, Lee TD, Légaré JF: Myocardial fibrosis in response to Angiotensin II is preceded by the recruitment of mesenchymal progenitor cells. Lab Invest 2011, 91:565-578

16. Sopel M, Falkenham A, Oxner A, Ma I, Lee TD, Legare JF: Fibroblast progenitor cells are recruited into the myocardium prior to the development of myocardial fibrosis. Int J Exp Pathol 2012, 93:115-124

17. Pinto AR, Paolicelli R, Salimova E, Gospocic J, Slonimsky E, Bilbao-Cortes D, Godwin JW, Rosenthal NA: An abundant tissue macrophage population in the adult murine heart with a distinct alternatively-activated macrophage profile. PLoS One 2012, 7:e36814

18. Buiting AM, Van Rooijen N: Liposome mediated depletion of macrophages: an approach for fundamental studies. J Drug Target 1994, 2:357-362

19. van Rooijen N: Liposomes for targeting of antigens and drugs: immunoadjuvant activity and liposome-mediated depletion of macrophages. J Drug Target 2008, 16:529-534

20. Wynn TA, Chawla A, Pollard JW: Macrophage biology in development, homeostasis and disease. Nature 2013, 496:445-455

21. Falkenham A, Sopel M, Rosin N, Lee TD, Issekutz T, Légaré JF: Early fibroblast progenitor cell migration to the AngII-exposed myocardium is not CXCL12 or CCL2 dependent as previously thought. Am J Pathol 2013, 183:459-469

22. Rosin NL, Falkenham A, Sopel MJ, Lee TD, Legare JF: Regulation and role of connective tissue growth factor in AngII-induced myocardial fibrosis. Am J Pathol 2013, 182:714-726

23. Rosin NL, Sopel M, Falkenham A, Myers TL, Legare JF: Myocardial migration by fibroblast progenitor cells is blood pressure dependent in a model of angII myocardial fibrosis. Hypertens Res 2012, 35: $449-456$

24. Sugino H, Ozono R, Kurisu S, Matsuura H, Ishida M, Oshima T, Kambe M, Teranishi Y, Masaki H, Matsubara H: Apoptosis is not increased in myocardium overexpressing type 2 angiotensin II receptor in transgenic mice. Hypertension 2001, 37:1394-1398

25. Combadiere C, Potteaux S, Gao JL, Esposito B, Casanova S, Lee EJ, Debre P, Tedgui A, Murphy PM, Mallat Z: Decreased atherosclerotic lesion formation in CX3CR1/apolipoprotein E double knockout mice. Circulation 2003, 107:1009-1016

26. van Rooijen N, Hendrikx E: Liposomes for specific depletion of macrophages from organs and tissues. Methods Mol Biol 2010, 605:189-203

27. Holt DJ, Chamberlain LM, Grainger DW: Cell-cell signaling in cocultures of macrophages and fibroblasts. Biomaterials 2010, 31: 9382-9394

28. Weischenfeldt J, Porse B: Bone marrow-derived macrophages (BMM): isolation and applications. CSH Protoc 2008. doi: 10.1101/pdb.prot5080

29. Heidt T, Courties G, Dutta P, Sager HB, Sebas M, Iwamoto Y, Sun Y, Da Silva N, Panizzi P, van der Lahn AM, Swirski FK, Weissleder R, Nahrendorf M: Differential contribution of monocytes to heart macrophages in steady-state and after myocardial infarction. Circ Res 2014, 115:284-295

30. Pinto AR, Godwin JW, Rosenthal NA: Macrophages in cardiac homeostasis, injury responses and progenitor cell mobilisation. Stem Cell Res 2014, 13:705-714

31. Gordon S, Taylor P: Monocyte and macrophage heterogeneity. Nat Rev Immunol 2005, 5:953-964

32. Gordon S, Martinez FO: Alternative activation of macrophages: mechanism and functions. Immunity 2010, 32:593-604

33. Gordon S, Plüddemann A: Tissue macrophage heterogeneity: issues and prospects. Semin Immunopathol 2013, 35:533-540

34. Gordon S: The macrophage: past, present and future. Eur J Immunol 2007, 37(Suppl 1):S9-S17

35. Nahrendorf M, Swirski FK, Aikawa E, Stangenberg L, Wurdinger T, Figueiredo JL, Libby P, Weissleder R, Pittet MJ: The healing myocardium sequentially mobilizes two monocyte subsets with divergent and complementary functions. J Exp Med 2007, 204:3037-3047 
36. van der Laan AM, Ter Horst EN, Delewi R, Begieneman MP, Krijnen PA, Hirsch A, Lavaei M, Nahrendorf M, Horrevoets AJ, Niessen HW, Piek JJ: Monocyte subset accumulation in the human heart following acute myocardial infarction and the role of the spleen as monocyte reservoir. Eur Heart J 2014, 35:376-385

37. Swirski FF, Nahrendorf M, Etzrodt M, Wildgruber M, CortezRetamozo V, Panizzi P, Figueiredo JL, Kohler RH, Chudnovskiy A, Waterman P, Aikawa E, Mempel TR, Libby P, Weissleder R, Pittet MJ: Identification of splenic reservoir monocytes and their deployment to inflammatory sites. Science 2009, 325:612-616

38. Nahrendorf M, Pittet MJ, Swirski FK: Monocytes: protagonists of infarct inflammation and repair after myocardial infarction. Circulation 2010, 121:2437-2445

39. Sosnovik DE, Nahrendorf M, Deliolanis N, Novikov M, Aikawa E, Josephson L, Rosenzweig A, Weissleder R, Ntziachristos V: Fluorescence tomography and magnetic resonance imaging of myocardial macrophage infiltration in infarcted myocardium in vivo. Circulation 2007, 115:1384-1391

40. Medina-Contreras O, Geem D, Laur O, Williams IR, Lira SA, Nusrat A, Parkos CA, Denning TL: CX3CR1 regulates intestinal macrophage homeostasis, bacterial translocation, and colitogenic Th17 responses in mice. J Clin Invest 2011, 121:4787-4795

41. Furuichi K, Gao JL, Murphy PM: Chemokine receptor CX3CR1 regulates renal interstitial fibrosis after ischemia-reperfusion injury. Am J Pathol 2006, 169:372-387

42. Wasmuth HE, Zaldivar MM, Berres ML, Werth A, Scholten D, Hillebrandt S, Tacke F, Schmitz P, Dahl E, Wiederholt T, Hellerbrand C, Berg T, Weiskirchen R, Trautwein C, Lammert F: The fractalkine receptor CX3CR1 is involved in liver fibrosis due to chronic hepatitis C infection. J Hepatol 2008, 48:208-215

43. Frisancho-Kiss S, Coronado MJ, Frisancho JA, Lau VM, Rose NR, Klein SL, Fairweather D: Gonadectomy of male BALB/c mice increases Tim-3(+) alternatively activated M2 macrophages, Tim-3(+) T cells, Th2 cells and Treg in the heart during acute coxsackievirus-induced myocarditis. Brain Behav Immun 2009, 23:649-657

44. Novak ML, Koh TJ: Macrophage phenotypes during tissue repair. J Leukoc Biol 2013, 93:875-881

45. Sunderkötter C, Nikolic T, Dillon MJ, Van Rooijen N, Stehling M, Drevets DA, Leenen PJ: Subpopulations of mouse blood monocytes differ in maturation stage and inflammatory response. J Immunol 2004, 172:4410-4417
46. Frantz S, Hofmann U, Fraccarollo D, Schäfer A, Kranepuhl S, Hagedorn I, Nieswandt B, Nahrendorf M, Wagner H, Bayer B, Pachel C, Schön MP, Kneitz S, Bobinger T, Weidemann F, Ertl G, Bauersachs J: Monocytes/macrophages prevent healing defects and left ventricular thrombus formation after myocardial infarction. FASEB J 2013, 27:871-881

47. van Amerongen MJ, Harmsen MC, van Rooijen N, Petersen AH, van Luyn MJ: Macrophage depletion impairs wound healing and increases left ventricular remodeling after myocardial injury in mice. Am J Pathol 2007, 170:818-829

48. Ben-Mordechai T, Holbova R, Landa-Rouben N, Harel-Adar T, Feinberg MS, Abd Elrahman IA, Blum G, Epstein F, Silman Z, Cohen S, Leor J: Macrophage subpopulations are essential for infarct repair with and without stem cell therapy. J Am Coll Cardiol 2013, 62:1890-1901

49. Bujak M, Frangogiannis NG: The role of TGF-beta signaling in myocardial infarction and cardiac remodeling. Cardiovasc Res 2007 , 74:184-195

50. Werner F, Jain MK, Feinberg MW, Sibinga NE, Pellacani A, Wiesel P, Chin MT, Topper JN, Perrella MA, Lee ME: Transforming growth factor-beta 1 inhibition of macrophage activation is mediated via Smad3. J Biol Chem 2000, 275:36653-36658

51. Harel-Adar T, Ben Mordechai T, Amsalem Y, Feinberg MS, Leor J, Cohen S: Modulation of cardiac macrophages by phosphatidylserinepresenting liposomes improves infarct repair. Proc Natl Acad Sci U S A 2011, 108:1827-1832

52. Arai M, Ikawa Y, Chujo S, Hamaguchi Y, Ishida W, Shirasaki F, Hasegawa M, Mukaida N, Fujimoto M, Takehara K: Chemokine receptors CCR2 and CX3CR1 regulate skin fibrosis in the mouse model of cytokine-induced systemic sclerosis. J Dermatol Sci 2013, 69:250-258

53. Ishida Y, Gao JL, Murphy PM: Chemokine receptor CX3CR1 mediates skin wound healing by promoting macrophage and fibroblast accumulation and function. J Immunol 2008, 180:569-579

54. Yona S, Kim KW, Wolf Y, Mildner A, Varol D, Breker M, StraussAyali D, Viukov S, Guilliams M, Misharin A, Hume DA, Perlman H, Malissen B, Zelzer E, Jung S: Fate mapping reveals origins and dynamics of monocytes and tissue macrophages under homeostasis. Immunity 2013, 38:79-91

55. Landsman L, Bar-On L, Zernecke A, Kim KW, Krauthgamer R, Shagdarsuren E, Lira SA, Weissman IL, Weber C, Jung S: CX3CR1 is required for monocyte homeostasis and atherogenesis by promoting cell survival. Blood 2009, 113:963-972 